\title{
The secondary stars in cataclysmic variables and low-mass X-ray binaries
}

\author{
D. A. Smith ${ }^{1,2 *}$ and V. S. Dhillon ${ }^{3 *}$ \\ ${ }^{1}$ Institute of Astronomy, Madingley Road, Cambridge CB3 OHA \\ ${ }^{2}$ Royal Greenwich Observatory, Madingley Road, Cambridge CB3 OEZ \\ ${ }^{3}$ Department of Physics and Astronomy, University of Sheffield, Sheffield S3 7RH
}

Accepted 1998 August 3. Received 1998 July 20; in original form 1998 March 18

\begin{abstract}
A B S T R A C T
We critically re-examine the available data on the spectral types, masses and radii of the secondary stars in cataclysmic variables (CVs) and low-mass X-ray binaries (LMXBs), using the new catalogue of Ritter \& Kolb as a starting point. We find there are 55 reliable spectral type determinations and only 14 reliable mass determinations of CV secondary stars (10 and 5, respectively, in the case of LMXBs). We derive new spectral type-period, mass-radius, mass-period and radius-period relations, and compare them with theoretical predictions. We find that $\mathrm{CV}$ secondary stars with orbital periods shorter than $7-8 \mathrm{~h}$ are, as a group, indistinguishable from main-sequence stars in detached binaries. We find that it not valid, however, to estimate the mass from the spectral type of the secondary star in CVs or LMXBs. We find that LMXB secondary stars show some evidence for evolution, with secondary stars which are slightly too large for their mass. We show how the masses and radii of the secondary stars in CVs can be used to test the validity of the disrupted magnetic braking model of $\mathrm{CV}$ evolution, but we find that the currently available data are not sufficiently accurate or numerous to allow such an analysis. As well as considering secondary star masses, we also discuss the masses of the white dwarfs in CVs, and find mean values of $\bar{M}_{1}=0.69 \pm 0.13 \mathrm{M}_{\odot}$ below the period gap, and $\bar{M}_{1}=0.80 \pm 0.22 \mathrm{M}_{\odot}$ above the period gap.
\end{abstract}

Key words: binaries: close - stars: late-type - novae, cataclysmic variables - X-rays: stars.

\section{INTRODUCTION}

Cataclysmic variables (CVs) are semidetached binary stars in which a white dwarf primary accretes material from a Roche lobe-filling secondary. For a thorough review of CVs see Warner (1995a). The spectral type and luminosity class of the secondary star can be estimated from basic theory, as follows. Kepler's third law can be written as

$\frac{4 \pi^{2} a^{3}}{G P^{2}}=M_{1}+M_{2}=M_{2}\left(\frac{1+q}{q}\right)$,

where $q=M_{2} / M_{1}$. The volume-equivalent radius of the Roche lobe can be approximated by

$\frac{R_{2}}{a}=0.47\left(\frac{q}{1+q}\right)^{1 / 3}$.

This relation is a slightly modified form of the Paczyński (1971) equation, and we have found that it is accurate to less than 3 per cent over the range of mass ratios relevant for CVs $(0.01<q<1.0)$. Combining equations (1) and (2) gives the mean density-period

*E-mail: dsmith@ast.cam.ac.uk (DAS); vik.dhillon@ sheffield.ac.uk (VSD) relation,

$\frac{\rho}{\rho_{\odot}}=\left(\frac{M_{2}}{\mathrm{M}_{\odot}}\right)\left(\frac{R_{2}}{\mathrm{R}_{\odot}}\right)^{-3}=75.5 P^{-2}(\mathrm{~h})$,

which is accurate to $\sim 6$ per cent (see Eggleton 1983).

Typical lower main-sequence mean densities range from $\sim 50 \rho_{\odot}$ for M8 dwarfs (Allen 1976), corresponding to the minimum orbital period of $\mathrm{CVs}$ around $80 \mathrm{~min}$, to $\sim 1 \rho_{\odot}$ for G0 dwarfs, corresponding to an orbital period of around $9 \mathrm{~h}$. At periods longer than $\sim 9 \mathrm{~h}$, the density of the secondary star must be subsolar, corresponding to F-type (and earlier) main-sequence stars. F-type main-sequence stars have masses above solar $\left(M \sim 1.3 \mathrm{M}_{\odot}\right.$ for an F5 dwarf, $M \sim 1.6 \mathrm{M}_{\odot}$ for an F0 dwarf, Gray 1992), which is relevant when considering the formal requirement of $q<5 / 6$ for stable, conservative mass transfer (e.g. Frank, King \& Raine 1992). Since the mass of the white dwarf must be below the Chandrasekhar limit, the secondary is forced to have $M_{2}<1.2 \mathrm{M}_{\odot}$. As the white dwarf population is biased towards much lower masses (the mean white dwarf mass in CVs is $0.77 \pm 0.21 \mathrm{M}_{\odot}$; see Section 6), the number of systems with secondary stars above solar mass is very small indeed. Secondary stars with subsolar mean densities in long-period CVs must therefore be low-mass evolved $\mathrm{M}$ or $\mathrm{K}$ stars rather than intermediate-mass main-sequence $\mathrm{F}$ stars. In summary, for the 
orbital period range in which most CVs lie $(1.3 \leqq P \leqq 9 \mathrm{~h})$, the secondary stars should be M, K or G main-sequence dwarfs, while longer period systems must harbour secondaries which have evolved away from the main sequence.

Even though we have just shown that most $\mathrm{CV}$ secondaries should have lower main-sequence densities, it is not clear that they should appear as lower main-sequence stars. This is because $\mathrm{CV}$ secondaries are subject to a number of extreme environmental factors to which field stars are not. Specifically, CV secondaries are:

(i) situated $\sim 1 \mathrm{R}_{\odot}$ from a hot, irradiating source (see Smith 1995);

(ii) rapidly rotating $\left(\sim 100 \mathrm{~km} \mathrm{~s}^{-1}\right)$;

(iii) Roche-lobe-shaped;

(iv) losing mass at a rate of $\sim 10^{-8}-10^{-11} \mathrm{M}_{\odot} \mathrm{yr}^{-1}$;

(v) survivors of a common-envelope phase during which they existed within the atmosphere of a giant star, and

(vi) exposed to nova outbursts every $\sim 10^{4} \mathrm{yr}$.

It is the purpose of this paper to see if the above environmental factors alter the gross properties (masses, radii, spectral types) of the secondary stars in CVs. This is not the first time that the question of whether CV secondaries lie on the main sequence has been investigated. Echevarría (1983) concluded that they did not. He came to this conclusion by calculating an empirical mass-radius relation for field stars and then using a density-period relation similar to equation (3) to calculate a mass-period relation. This was then combined with a spectral type-mass relation to get a spectral type-period relation for field stars. By comparing this relation with the measured spectral types and periods of $17 \mathrm{CVs}$ he found that the CVs did not fit his relation, and concluded that they were therefore not on the main sequence.

Warner (1995a,b) disputed Echevarría's conclusion by arguing that he had equated a poorly fitting power law to the mass-spectral type relationship and that, as a result of this, Echevarría's derived relationship systematically predicted spectral types that were too early for long-period CVs and too late for the short-period CVs. Instead, Warner plotted the CV secondary stars on a spectral typeperiod diagram and calculated equivalent periods of field stars using a mass-period relation, derived using a mass-radius relation which fits both the CV data of Webbink (1990) and the field star data of Popper (1980). Warner concluded that, as a group, secondary stars have masses, radii and spectral types related in exactly the same way as main-sequence stars, although some individual CVs do depart from the average properties. A similar conclusion has been reached by Ritter (1983) and Patterson (1984).

Low mass X-ray binaries (LMXBs) are also semidetached systems, the difference being that the primary in these systems is a neutron star or a black hole. The secondary stars in LMXBs should be similar to those in $\mathrm{CVs}$, except that they are irradiated to a much greater degree by X-rays. Also, the formation of LMXBs containing neutron stars requires that the secondary stars have a mass between $1.3-1.5 \mathrm{M}_{\odot}$ at the onset of mass transfer, and that they should be significantly nuclear-evolved (King \& Kolb 1997). LMXB secondaries should therefore appear even less like main-sequence stars than their CV counterparts.

In this paper we repeat the analyses of Echevarría (1983), Ritter (1983), Patterson (1984), Webbink (1990) and Warner (1995a,b), and go a few steps further, using the more extensive data set now available (Ritter \& Kolb 1998 and other sources) to derive the first reliable spectral type-period relation for CVs. We present new mass-radius, mass-period and radius-period relations for $\mathrm{CV}$ secondary stars with reliable system parameters, and compare the parameters of the CV and LMXB secondaries with those of detached binaries as listed in the reviews of Popper (1980) and Andersen (1991). We also briefly examine the observed mass distribution of the white dwarf population in CVs, and conclude with a discussion on how the measured masses and radii of $\mathrm{CV}$ secondary stars can be used to constrain the disrupted magnetic braking model of $\mathrm{CV}$ evolution.

\section{THE ORBITAL PERIOD-SPECTRAL TYPE RELATION}

The spectral type of the secondary star has been measured in $66 \mathrm{CVs}$ with known orbital periods, according to the catalogue of Ritter \& Kolb (1998). A number of these measurements, however, are dubious, relying on main-sequence assumptions, or infrared colours which are contaminated by disc emission (Berriman, Szkody \& Capps 1985), to determine the spectral types. The only reliable method of determining spectral types is to detect absorption features in the spectra of the secondary stars. One can then compare the equivalent widths of the secondary star absorption features with those of isolated dwarf stars (e.g. Wade \& Horne 1988), or simply match the observed absorption features with those in a set of template spectra, either by eye (e.g. Friend et al. 1990a,b) or by using an optimal subtraction technique (e.g. Smith, Dhillon \& Marsh 1998). Absorption features have been detected (and hence reliable spectral types determined) in 55 of the $66 \mathrm{CVs}$ catalogued by Ritter \& Kolb (1998), as listed in Table 1. Most of the spectral types listed in Table 1 do not have error bars associated with them, so we have instead assigned a weighting to each determination. In most cases, a weighting of 1 implies a careful spectral type determination using ample template dwarfs, and a weighting of 0.5 implies that there is a larger uncertainty in the exact value of the spectral type, often as a result of insufficient template dwarfs having been observed.

The spectral types of the secondary stars listed in Table 1 are plotted against orbital period in Fig. 1. Two linear least-squares fits were performed, one above and one below the kink at an orbital period of $4 \mathrm{~h}$. Note that we omitted seven CVs from the fit (indicated by the asterisks in Table 1) which are believed to harbour evolved secondary stars. For the sake of simplicity, we have treated each spectral type as being divided into 10 equal subtypes. ${ }^{1}$ The resulting fits are given by:

$$
\begin{aligned}
& S p(2)=26.5-0.7 P, \quad P<4 \mathrm{~h} \\
& \pm 0.7 \pm 0.2 \\
& =\quad 33.2-2.5 P, \quad P>4 \mathrm{~h} \\
& \pm 3.1 \pm 0.5 \text {, }
\end{aligned}
$$

where $\operatorname{Sp}(2)=0$ represents a spectral type G0, $\operatorname{Sp}(2)=10$ is $\mathrm{K} 0$, and $\operatorname{Sp}(2)=20$ is M0. The rms scatter is 0.8 spectral subtypes for $P<4$ h, and 3.0 subtypes for $P>4$ h.

The solid lines in the spectral type-period diagram represent our two-part linear fit to the CVs (equation 4), while the dashed line represents Echevarría's equation. Also plotted as a comparison in Fig. 1 are the Sun and 49 other late-type main-sequence stars in

\footnotetext{
${ }^{1}$ The MK system officially does not contain the $\mathrm{K} 8$ or $\mathrm{K} 9$ subtypes, nor those of M4.5, M5.5, etc. (Jaschek \& Jaschek 1987). However, these are in (infrequent) use, so to make the spectral type-period relation simple to use, and since the system is in any case non-linear, we have adopted a system where each spectral type is divided into 10 subtypes. This system was also followed by Echevarría (1983) and Warner (1995b) in their spectral typeperiod diagrams.
} 
Table 1. Spectral types of secondary stars and orbital periods (in h) of cataclysmic variables. Key: $\mathrm{CN}=$ classical nova; $\mathrm{DN}=\mathrm{dwarf}$ nova; $\mathrm{RN}=$ recurrent nova; $\mathrm{NL}=$ nova-like; $\mathrm{P}=$ polar; $\mathrm{IP}=$ intermediate polar; ${ }^{*}=$ systems with "evolved" secondary stars, omitted from the linear fit in Fig. 1.

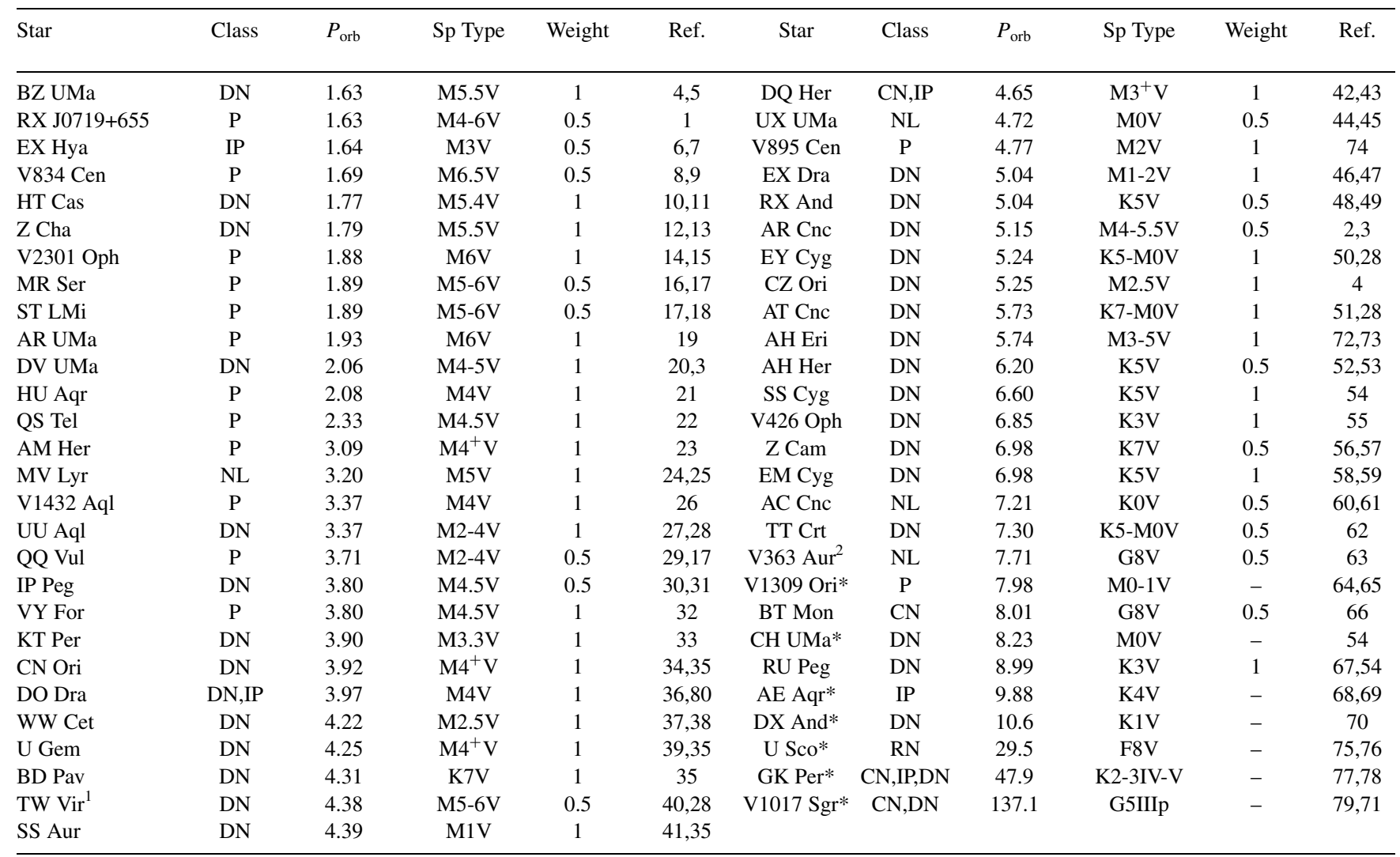

References: $\quad$ 1. Tovmassian et al. 1997, 2. Howell et al. 1990, 3. Mukai et al. 1990, 4. Ringwald, Thorstensen \& Hamwey 1994,

5. Jurcevic et al. 1994, 6. Sterken et al. 1983, 7. Dhillon et al. 1997, 8. Schwope et al. 1993, 9. Puchnarewicz et al. 1990,

10. Horne, Wood \& Steining (1991), 11. Marsh 1990, 12. Robinson et al. 1995, 13. Wade \& Horner 1988,

14. Barwig, Ritter \& Baernbantner 1994, 15. Silber et al. 1994, 16. Schwope et al. 1991, 17. Mukai \& Charles 1986,

18. Cropper 1986, 19. Remillard, Schachter \& Silber 1994, 20. Howell et al. 1988, 21. Glenn et al. 1994,

22. Schwope et al. 1995, 23. Young, Schneider \& Schectman 1981, 24. Skillman, Patterson \& Thorstensen 1995 ,

25. Schneider, Young \& Schectman 1981, 26. Watson et al. 1995, 27. Ritter \& Kolb 1998, 28. Smith et al. 1997 ,

29. Andronov \& Fuhrmann 1987, 30. Wolf et al. 1993, 31. Martin, Jones \& Smith 1987, 32. Beuermann et al. 1989,

33. Thorstensen \& Ringwald 1997, 34. Barrera \& Vogt 1989, 35. Friend et al. 1990a, 36. Haswell et al. 1997,

37. Ringwald et al. 1996, 38. Hawkins, Smith \& Jones 1990, 39. Smak 1993, 40. Shafter 1983, 41. Shafter \& Harkness 1986,

42. Zhang et al. 1995, 43. Young \& Schneider 1981, 44. Baptista et al. 1995, 45. Rutten et al. 1994, 46. Fiedler 1994,

47. Billington, Marsh \& Dhillon 1996, 48. Kaitchuck 1989, 49. Dhillon \& Marsh 1995, 50. Sarna, Pych \& Smith 1995,

51. Goetz 1986, 52. Horne, Wade \& Szkody 1986b, 53. Bruch 1987, 54. Friend et al. 1990b, 55. Hessman 1988,

56. Thorstensen \& Ringwald 1995, 57. Szkody \& Wade 1981, 58. Stover, Robinson \& Nather 1981,

59. Beuermann \& Pakull 1984, 60. Okazaki, Kitamura \& Yamasaki 1982, 61. Schlegel, Kaitchuck \& Honeycutt 1984,

62. Szkody et al. 1992, 63. Schlegel, Honeycutt \& Kaitchuck 1986, 64. Buckley \& Shafter 1995, 65. Shafter et al. 1995,

66. Smith, Dhillon \& Marsh (1998), 67. Stover 1981b, 68. Welsh, Horne \& Gomer 1993, 69. Casares et al. 1996,

70. Drew, Jones \& Woods 1993, 71. Sekiguchi 1992, 72. Thorstensen 1997, 73. Howell, Liebert \& Mason 1994,

74. Stobie et al. 1996, 75. Schaefer \& Ringwald 1995, 76. Johnston \& Kulkarni 1992, 77. Reinsch 1994,

78. Crampton, Cowley \& Fisher 1986, 79. Kraft 1964, 80. Mateo, Szkody \& Garnavich 1991.

${ }^{1}$ Spectral type uncertain since it was determined from a spectrum taken while the star was on the rise to outburst.

${ }^{2}$ Spectral type given as late G.

detached binaries. 12 of these 49 have been taken from the list of Andersen (1991), while the review paper by Popper (1980) lists a further 37 stars with less well-refined mass and radius measurements, but which are suitable for comparison purposes; we have taken 11 stars from Popper's table 2 (detached main-sequence binaries, B6 to M), six stars from his table 7 (resolved spectroscopic binaries), and 20 from his table 8 (visual binaries).

The stars in detached binaries are plotted in Fig. 1, not according to their actual orbital periods, but rather according to the period of a
CV containing a Roche lobe-filling secondary star of that mass and radius. This assumes, therefore, that the spectral types of the stars in detached binaries would remain unchanged if they were to become lobe-filling secondaries. There are two ways of performing the conversion from mass and radius to period, depending on how accurately the radii of the detached stars are known. In the case of the stars listed by Popper, which have poorly determined radii, we have converted from mass to period using the empirical massperiod relation we derive in Section 5 (equation 8). Typical errors in 


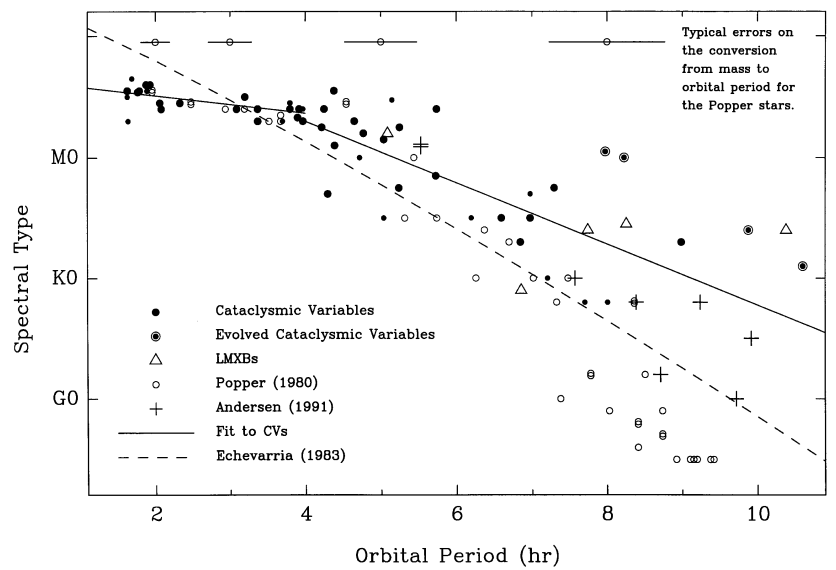

Figure 1. The spectral types of the secondary stars in CVs and LMXBs versus their orbital periods. The CVs have been plotted as filled circles, large circles denoting better spectral type determinations (weight $=1$ in Table 1 ) than the small circles (weight $=0.5$ ). LMXBs are denoted by triangles. Also plotted are 50 isolated stars for which the spectral types and masses have been measured (see text for details). The solid line is a two-part linear fit to the CVs, given by equation (4). The dashed line is the relationship derived by Echevarría (1983).

this conversion, as calculated from the errors in equation (8), are plotted for different orbital periods at the top of Fig. 1. In the case of the stars listed by Andersen, all of which are eclipsing binaries and hence have accurately determined radii, we have used equation (3) directly to convert from mass and radius to period. These have a much smaller associated error ( 3 per cent), so the crosses in Fig. 1 (Andersen's points) should be given greater weighting than the open circles (Popper's points) when interpreting the diagram.

There appears to be little difference between the distributions of the CVs and the main-sequence stars in Fig. 1 for periods below $4 \mathrm{~h}$. Above $4 \mathrm{~h}$, there is much larger scatter in the two distributions but they still seem to agree up to 7-8 $\mathrm{h}$. Beyond $7-8 \mathrm{~h}$ the two distributions show a definite divergence and some evolved secondary stars appear, while beyond $9 \mathrm{~h}$ the secondary stars all show signs of evolution (as is to be expected, see Section 1). Echevarría's relationship is a poor fit to the detached systems, as noted by Warner (1995a,b), and can be seen to erroneously predict later spectral types at short periods, and earlier spectral types at long periods, than those which have been observed. Note that the Echevarría relationship was used as the basis for the claim by Friend et al. (1990a,b) that the secondary stars in CVs were 'too cool for comfort' and 'too cool for credibility,' which no longer appears to be true (but see Section 4).

In Fig. 1, we have also plotted the five low-mass X-ray binaries (LMXBs) with known orbital periods (below $12 \mathrm{~h}$ ) and spectral types. They are listed in Table 2. To within the scatter, the LMXBs appear to have the same distribution as the CVs, indicating that LMXBs with orbital periods of $7-8 \mathrm{~h}$ and longer harbour evolved secondary stars.

\section{THE MASS-RADIUS RELATION}

The masses of the component stars in CVs can be determined in a number of different ways, using measurements of the following.

(i) The radial velocity semi-amplitude of the white dwarf, $K_{\mathrm{W}}$. This is inferred from the velocity variations of the wings of the emission lines, which arise in the inner accretion regions and are assumed to follow the motion of the white dwarf. These measurements are often unreliable because of contamination from other emission-line sources, such as the bright spot and the irradiated face of the secondary star, which do not follow the motion of the white dwarf and hence introduce phase shifts in the radial velocity curves. It is possible to correct for this contamination using diagnostic diagrams (Shafter, Szkody \& Thorstensen 1986), light centre diagrams (Marsh 1988) and symmetry analyses of Doppler tomograms (Still 1996). However we do not accept that any of these methods do give truly reliable values of $K_{\mathrm{W}}$ because of the uncertainty in the extrapolation needed to correct for the large phase shifts in the radial velocity curves.

(ii) The radial velocity semi-amplitude of the secondary star, $K_{\mathrm{R}}$. This is measured using the motion of absorption lines such as the NaI 8190- $\AA$ doublet (e.g. Friend et al. 1990a,b) or by using skew mapping (Smith et al. 1998). Another option is to follow the motion of the secondary star using the line emission from its irradiated inner face (e.g. Beuermann \& Thomas 1990). All of these $K_{\mathrm{R}}$ measurements are subject to errors due to the non-uniform distribution of the emission/absorption line strength on the surface of the secondary star, although this can be corrected for (e.g. Wade \& Horne (1988); Rutten \& Dhillon 1994).

Table 2. Spectral types, masses and radii of secondary stars in LMXBs.

\begin{tabular}{|c|c|c|c|c|c|c|}
\hline Star & $P_{\text {orb }}(\mathrm{hr})$ & Spectral Type & $M_{1}\left(\mathrm{M}_{\odot}\right)$ & $M_{2}\left(\mathrm{R}_{\odot}\right)$ & $R_{2}\left(\mathrm{R}_{\odot}\right)$ & References \\
\hline V518 Per (GRO J0422+32, Nova Per 1992) & 5.09 & $\mathrm{M} 2 \pm 2 \mathrm{~V}$ & & & & 1,2 \\
\hline MM Vel (Nova Vel 1993) & 6.86 & early K & & & & 3 \\
\hline V616 Mon (A0620-00) & 7.75 & $\mathrm{~K} 4 \mathrm{~V}$ & $3.89-4.12$ & $0.19-0.32$ & $0.53-0.63$ & 4,5 \\
\hline QZ Vul (GS 2000+25) & 8.26 & K3-6V & $6.04-13.9$ & $0.26-0.59$ & $0.62-0.81$ & 6 \\
\hline GU Mus (Nova Mus 1991) & 10.4 & $\mathrm{~K} 3-4 \mathrm{~V}$ & $6.98 \pm 1.45$ & $0.94 \pm 0.40$ & $1.06 \pm 0.15$ & $7,8,9$ \\
\hline V2107 Oph (Nova Oph 1977) & 12.5 & $\mathrm{~K} 5 \mathrm{~V}$ & & & & 10 \\
\hline V822 Cen (Cen X-4) & 15.1 & $\mathrm{~K} 5 \mathrm{~V}$ & & & & 11,12 \\
\hline V1333 Aql (Aq1 X-1) & 19.0 & $\mathrm{~K} 5 \mathrm{~V}$ & & & & 11 \\
\hline V1033 Sco (GRO J1655-40, Nova Sco 1994) & 62.9 & F3-6IV & $7.02 \pm 0.22$ & $2.34 \pm 0.12$ & $4.85 \pm 0.08$ & 13 \\
\hline V404 Cyg (GS 2023+338) & 155.3 & K0IV & $12_{-2}^{+3}$ & $0.7_{-0.2}^{+0.3}$ & $6.0_{-0.5}^{+0.7}$ & 14,15 \\
\hline
\end{tabular}

References: 1. Chevalier \& Ilovaisky 1996, 2. Casares et al. 1995, 3. Shahbaz et al. 1996b, 4. McClintock \& Remillard 1986,

5. Marsh, Robinson \& Wood 1994, 6. Harlaftis, Horne \& Filippenko 1996, 7. Orosz et al. 1996, 8. Casares et al. 1997,

9. This paper. 10. Harlaftis et al. 1997, 11. Shahbaz, Naylor \& Charles 1996c, 12. McClintock \& Remillard 1990,

13. Orosz \& Bailyn 1997, 14. Casares \& Charles 1994, 15. Shahbaz et al. 1994b. 
(iii) The projected rotational velocity of the secondary star, $V \sin i$. This is usually measured through comparison with (slowly rotating) field star template spectra which are given a series of artificial rotational broadenings. A constant times each template spectrum is then subtracted from the object spectrum to give a residual spectrum. The template which yields the smoothest residual provides the value of $V \sin i$ (e.g. Marsh, Robinson \& Wood 1994; Smith et al. 1998). An alternative method of measuring $V \sin i$ is that of cross-correlating with a template spectrum and measuring the width of the cross-correlation peak, $\sigma_{\text {rot }}$ (e.g. Horne, Wade \& Szkody 1986b). Models of rotating stars with various amounts of limb darkening are then used to find values of $V \sin i$ corresponding to $\sigma_{\text {rot }}$. Measurement of $V \sin i$ provides a powerful constraint on $M_{2}$, as the relation between them is only weakly dependent on $q$ (see equation B5 in Appendix B).

(iv) The full width of the eclipse at half depth, $\Delta \phi_{1 / 2}$. At half depth it is assumed that one half of the accretion disc is eclipsed, corresponding to the point at which the white dwarf is eclipsed. This ceases to be true if the disc is asymmetrical, e.g., if there is a dominant bright spot, or if there is no disc, e.g., in magnetic CVs. If the system is non-magnetic and does not have a dominant bright spot, then the measurement of $\Delta \phi_{1 / 2}$ provides a relationship between $q$ and the inclination $i$ (e.g. Smith et al. 1998).

(v) The duration and phase of the ingress/egress of the bright spot. This assumes that the bright spot lies on a ballistic trajectory from the $L_{1}$ point, i.e., there is no significant magnetic field. This provides another relationship between $q$ and $i$ (e.g. Wood et al. 1989).

(vi) The duration of the ingress/egress of the white dwarf, $\Delta \phi_{\mathrm{wd}}$. This is a measure of the radius of the primary as a function of $i$ and the separation $a$. The presence of an extended boundary layer can, however, cause the radius of the white dwarf to be overestimated and hence the mass to be underestimated (e.g. Wood et al. 1989).

(vii) The radius of the orbit of the white dwarf about the centre of mass, $a_{\mathrm{wd}}$, for rapidly rotating white dwarfs. This can be estimated from the spin pulse delay (e.g., AE Aqr; Eracleous et al. 1994). The only question is whether the white dwarf is the source of the pulses; in AE Aqr the spin pulse delay is exactly in phase with the white dwarf, so there is little doubt.

(viii) The ellipsoidal variations due to the changing aspect of the distorted Roche lobe-filling secondary star. These are particularly prominent in the infrared, showing up as a distinctive doublehumped modulation in the light curve. With appropriate modelling it is possible to constrain the inclination using ellipsoidal variations. This technique is especially useful for non-eclipsing systems (e.g. Hilditch 1995). The main uncertainty with this technique is the contribution of the disc to the total flux, which reduces the amplitude of the variations, resulting in an underestimate of the inclination. This can be corrected for if the secondary star has been detected spectroscopically (e.g. Shahbaz et al. 1996a).

(ix) Linear polarization light curves of magnetic CVs. These can be modelled to constrain $i$ (e.g. Wickramasinghe et al. 1991).

(x) The width of the base of the emission lines. This gives the projected rotational velocity of the innermost parts of the accretion disc, and hence a relation between $M_{1}, R_{1}$ and $i$ (e.g. Kuerster \& Barwig 1988). This quantity is, however, difficult to measure with any precision.

(xi) The separation of the two peaks in the emission lines, $v_{\mathrm{D}}$. This is used to relate the accretion disc radius (which must be less than the radius of the primary star's Roche lobe) and the mass of the primary star (e.g. Horne, Verbunt \& Schneider 1986a). In combination with $K_{\mathrm{R}}$, this can provide an upper limit to $q$. (xii) The orbital modulations of $V \sin i$. With several assumptions about limb and gravity darkening these modulations can be modelled to constrain the inclination (e.g. Casares et al. (1996); Shahbaz 1998). This technique is really a subset of mass derivations using Roche tomography (Rutten \& Dhillon 1996), which utilize the modulations in line strength and position as well as width to determine the component masses.

(xiii) A mass-radius relation for the white dwarf (e.g. Hamada \& Salpeter (1961); Nauenberg 1972). This is commonly used in conjunction with item (vi).

(xiv) A main-sequence mass-radius relation for the secondary star. The assumption of a main-sequence mass-radius relation is made frequently in the literature, and it is the validity of this assumption which we wish to test.

Of these various measurements and techniques for mass determination, our preferred method is the combination of $K_{\mathrm{R}}, V \sin i$ and $\Delta \phi_{1 / 2}$, or if the system is non-eclipsing, ellipsoidal variations. These parameters are simple to measure if the secondary is sufficiently bright, do not depend on any assumptions, other than that the secondary fills its Roche lobe, and can have any biases (due to irradiation of the secondary, for example) corrected for quite straightforwardly (e.g. Davey \& Smith 1996).

Ritter \& Kolb (1998) list values for the mass of the secondary star in $81 \mathrm{CVs}$. The vast majority of these mass determinations, however, have been derived using a main-sequence mass-radius relation. As our goal is the derivation of a new mass-radius relation for CV secondary stars, we have been forced to omit from our consideration all of the mass estimates which make a mainsequence assumption. This leaves only 20 mass determinations. Of these, five use the width of the emission lines ( $x$ and $x i$ ), which we believe to be extremely unreliable due to measurement uncertainties, two use the eclipse method (iv,v and vi) when it is clear from the shape of the light curve that this is invalid or at least unreliable, and six use determinations of $K_{\mathrm{W}}$ which are derived from radial velocity curves with significant phase shifts and hence do not represent the motion of the white dwarf. In fact, the only mass determination wholly dependent on a $K_{\mathrm{W}}$ measurement which we have not rejected is that of IP Peg, as Marsh (1988) was able successfully to correct for the phase shift in the radial velocity curve using the light centres method.

The above filtering process leaves us with only seven reliable CV mass determinations listed in the Ritter \& Kolb (1998) catalogue. We have also uncovered a few additional mass determinations which are not listed in their catalogue. These are BT Mon, V895 Cen and V2051 Oph. We have also discovered a number of $\mathrm{CVs}$ in the literature which possess measurements of $K_{\mathrm{R}}, V \sin i$, $\Delta \phi_{1 / 2}$ and $i$, but which have had no mass calculations - for these objects (BD Pav, DX And, EX Dra, AM Her and the LMXB GU Mus), we performed Monte Carlo simulations similar to those described by Smith et al. (1998) to calculate the system parameters - these are listed in Appendix C.

In summary, we have 14 reliable $\mathrm{CV}$ mass determinations and eight less reliable mass determinations, the latter depending on sometimes dubious $K_{\mathrm{W}}$ measurements, or else the uncertain assumption that the eclipse method is valid (UU Aqr). The masses and radii are listed in Table 3 , and described in greater detail in Appendix A. Note also that a number of authors quoted only the masses of the secondary stars and not their radii - we have calculated the radii using equation (3). Where this is the case, the errors on the radii have been taken to be a third of the percentage errors on the mass (from equation 3 ). 
Table 3. Masses and radii of cataclysmic variables. Key: $\mathrm{DN}=$ dwarf nova; $\mathrm{CN}=$ classical nova; $\mathrm{NL}=$ nova-like; $\mathrm{P}=$ polar; $\mathrm{IP}=$ intermediate polar. Asterisks denote those stars whose system parameters were wholly dependent on $K_{\mathrm{W}}$ measurements derived from radial velocity curves exhibiting significant phase shifts.

\begin{tabular}{|c|c|c|c|c|c|c|c|}
\hline Star & Class & $P_{\text {orb }}$ & $M_{1}$ & $q=M_{2} / M_{1}$ & $M_{2}$ & $R_{2}$ & Refs \\
\hline V2051 Oph & DN & 1.50 & $0.78 \pm 0.06$ & $0.19 \pm 0.03$ & $0.15 \pm 0.03$ & $0.16 \pm 0.01$ & 1 \\
\hline OY Car & DN & 1.51 & $0.685 \pm 0.011$ & $0.102 \pm 0.003$ & $0.070 \pm 0.002$ & $0.127 \pm 0.002$ & 2 \\
\hline EX Hya* & IP & 1.64 & $0.49 \pm 0.03$ & $0.19 \pm 0.03$ & $0.095 \pm 0.013$ & $0.15 \pm 0.01$ & $3,4,5$ \\
\hline HT Cas & DN & 1.77 & $0.61 \pm 0.04$ & $0.15 \pm 0.03$ & $0.09 \pm 0.02$ & $0.154 \pm 0.013$ & 6 \\
\hline Z Cha & DN & 1.79 & $0.84 \pm 0.09$ & $0.20 \pm 0.02$ & $0.125 \pm 0.014$ & $0.172 \pm 0.010$ & 7,8 \\
\hline ST LMi & $\mathrm{P}$ & 1.89 & $0.76 \pm 0.30$ & $0.23 \pm 0.05$ & $0.17 \pm 0.07$ & $0.20 \pm 0.03$ & $5,9,10$ \\
\hline AM Her & $\mathrm{P}$ & 3.09 & $0.44 \pm 0.12$ & $0.64 \pm 0.10$ & $0.29 \pm 0.10$ & $0.33 \pm 0.04$ & $5,11,12,13$ \\
\hline IP Peg & DN & 3.80 & $1.15 \pm 0.10$ & $0.59 \pm 0.04$ & $0.67 \pm 0.08$ & $0.501 \pm 0.024$ & 14,15 \\
\hline CN Ori* & DN & 3.92 & $0.74 \pm 0.1$ & $0.66 \pm 0.04$ & $0.49 \pm 0.08$ & $0.46 \pm 0.03$ & 16 \\
\hline UU Aqr* & NL & 3.93 & $0.67 \pm 0.14$ & $0.33 \pm 0.10$ & $0.20 \pm 0.07$ & $0.34 \pm 0.04$ & 17 \\
\hline U Gem* & DN & 4.25 & $1.26 \pm 0.12$ & $0.46 \pm 0.03$ & $0.57 \pm 0.07$ & $0.510 \pm 0.023$ & 16,18 \\
\hline BD Pav* & DN & 4.30 & $0.95 \pm 0.10$ & $0.44 \pm 0.06$ & $0.43 \pm 0.10$ & $0.46 \pm 0.04$ & $5,16,19$ \\
\hline DQ Her & $\mathrm{CN}, \mathrm{IP}$ & 4.65 & $0.60 \pm 0.07$ & $0.66 \pm 0.04$ & $0.40 \pm 0.05$ & $0.49 \pm 0.02$ & 20,21 \\
\hline IX Vel & NL & 4.65 & $0.80_{-0.11}^{+0.16}$ & $0.65 \pm 0.04$ & $0.52_{-0.07}^{+0.10}$ & $0.530 \pm 0.025$ & 22 \\
\hline V895 Cen & $\mathrm{P}$ & 4.77 & $0.93 \pm 0.17$ & $0.51 \pm 0.12$ & $0.48 \pm 0.17$ & $0.51 \pm 0.06$ & 23 \\
\hline EX Dra & DN & 5.04 & $0.70 \pm 0.10$ & $0.84 \pm 0.12$ & $0.59 \pm 0.12$ & $0.59 \pm 0.04$ & $5,24,25$ \\
\hline EM Cyg* & DN & 6.98 & $0.56 \pm 0.05$ & $1.35 \pm 0.16$ & $0.76 \pm 0.10$ & $0.79 \pm 0.04$ & 5,26 \\
\hline AC Cnc* & NL & 7.21 & $0.82 \pm 0.13$ & $1.24 \pm 0.08$ & $1.02 \pm 0.14$ & $0.92 \pm 0.05$ & 27,28 \\
\hline V363 Aur* & NL & 7.71 & $0.86 \pm 0.08$ & $0.89 \pm 0.03$ & $0.77 \pm 0.04$ & $0.83 \pm 0.04$ & 29 \\
\hline BT Mon & $\mathrm{CN}$ & 8.01 & $1.04 \pm 0.06$ & $0.84 \pm 0.04$ & $0.87 \pm 0.06$ & $0.89 \pm 0.02$ & 30 \\
\hline $\mathrm{AE}$ Aqr & IP & 9.88 & $0.79 \pm 0.16$ & $0.630 \pm 0.012$ & $0.50 \pm 0.10$ & $0.86 \pm 0.06$ & $5,31,32$ \\
\hline DX And & DN & 10.60 & $0.51 \pm 0.12$ & $0.98 \pm 0.10$ & $0.50 \pm 0.14$ & $0.92 \pm 0.08$ & $5,33,34$ \\
\hline
\end{tabular}

References: 1. Baptista et al. 1998, 2. Wood et al. 1989, 3. Hellier 1996, 4. Sterken et al. 1983, 5. This paper, 6. Horne, Wood \& Steining 1991, 7. Wade \& Horner 1988, 8. Robinson et al. 1995, 9. Shahbaz \& Wood 1996, 10. Cropper 1986, 11. Davey* Smith 1996, 12. Southwell et al., 13. Wickramasinghe et al. 1991,

14. Martin et al. 1989, 15. Barrera \& Vogt 1989, 16. Friend et al. 1990a, 17. Baptista, Steiner \& Cieslinski 1994, 18. Smak 1993, 19. Harrop-Allin \& Warner 1996, 20. Zhang et al. 1995, 21. Horner, Welsh \& Wade 1993, 22. Beuermann \& thomas 1990, 23. Buckley et al., in preparation, 24. Billington, Marsh \& Dhillon 1996, 25. Fiedler, Barwig \& Mantel 1997, 26. Stover, Robinson \& Nather 1981, 27. Okazaki, Kitamura \& Yamasaki 1982, 28. Schlegel, Kaitchuck \& Honeycutt 1984, 29. Schlegel, Honeycutt \& Kaitchuck 1986, 30. Smith, Dhillon \& Marsh 1998, 31. Casares et al. 1996, 32. Welsh, Horne \& Gomer 1993, 33. Drew, Jones \& Woods 1993, 34. Hilditch 1995.

There are just five LMXBs listed by Ritter \& Kolb (1998; see also Beekman et al. 1997) which have reliable mass determinations. We have determined the system parameters of GU Mus using values of $V \sin i$ and $K_{\mathrm{R}}$ provided by Casares et al. (1997; who determined only upper and lower limits to the masses). The masses and radii of the LMXB secondary stars are listed in Table 2 and described in more detail in Appendix A.

Those CVs with reliable mass determinations have been plotted as filled circles in Fig. 2, while those which use dubious $K_{\mathrm{W}}$ determinations are ringed. The points representing DX And and AE Aqr are also ringed, as they have evolved secondary stars (Casares et al. 1996; Drew, Jones \& Woods 1993). LMXBs are plotted as open triangles. Open circles represent the masses and radii of the 50 isolated stars listed by Popper (1980) and, Andersen (1991) the latter having smaller error bars than the former.

We performed least-squares power-law and linear fits to the CVs using the formal error bars quoted in the literature and listed in Table 3. We have only fitted to the unringed, filled circles in Fig. 2, i.e., DX And, AE Aqr and those systems which have dubious $K_{\mathrm{W}}$ measurements have been omitted from the fits. The power-law fit is plotted as a solid line in the upper panel, and is given by

$\frac{R}{\mathrm{R}_{\odot}}=(0.91 \pm 0.09)\left(\frac{M}{\mathrm{M}_{\odot}}\right)^{(0.75 \pm 0.04)}$.

The linear fit is plotted as a dashed line in the upper panel, and is given by

$\frac{R}{\mathrm{R}_{\odot}}=(0.93 \pm 0.09)\left(\frac{M}{\mathrm{M}_{\odot}}\right)+(0.06 \pm 0.03)$.

We have also plotted the mass-radius relation of Warner (1995a,b),

$R=M^{13 / 15}$,

in the upper panel of Fig. 2 as a dotted line, which is an approximate fit to the data set given by Webbink (1990). Of these three massradius relations (equations 5,6 and 7), we would recommend the use of our equation (5), which has been fitted to our data using a leastsquares fitting procedure (as opposed to equation 7 , where Warner fitted the Webbink's data by eye, forcing the multiplicative constant to be unity and the power to be a simple ratio).

In the lower panel of Fig. 2, the dashed line is the theoretical lower main sequence of Chabrier \& Baraffe (1997), calculated using detailed models with the latest input physics, and the thin; solid line is the empirical mass-radius relation obtained from a volume-limited sample of nearby $\mathbf{M}$ dwarfs by Clemens et al. (1998; see also Reid \& Gizis 1997). The thick solid line shows the masses and radii of a secondary star in an evolutionary sequence computed by Kolb \& Baraffe (in preparation), again using the most up-to-date stellar input physics for low-mass stars and brown dwarfs currently available. This particular sequence uses a white dwarf mass of $M_{1}=0.7 \mathrm{M}_{\odot}$ and an initial secondary star mass of 

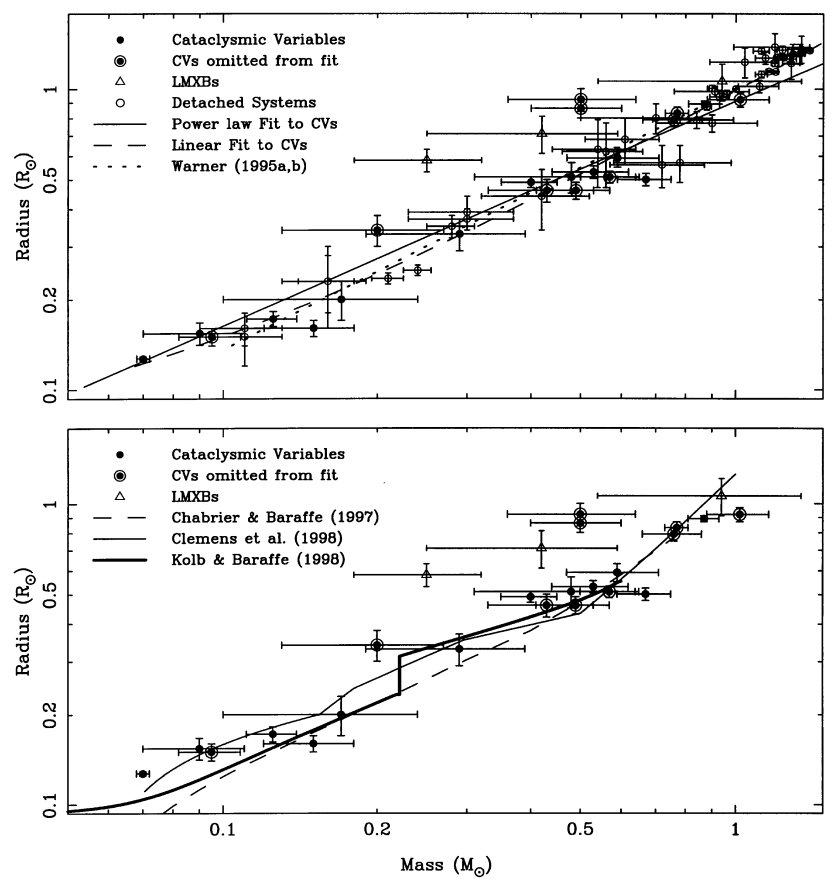

Figure 2. The masses and radii of the secondary stars in CVs and LMXBs. The upper panel shows a power-law fit (solid line) and a linear fit (dashed line) to the $\mathrm{CV}$ data (unringed, filled circles). The $\mathrm{CV}$ points which are ringed have been omitted from the fit. LMXBs are denoted by open triangles. Also plotted in the upper panel is the mass-radius relation (equation 7) derived by Warner (1995a,b). The lower panel shows the same data points as the upper panel, along with the theoretical models of Chabrier \& Baraffe (1997, the dashed line) and the empirical relation derived by Clemens et al. (1998, the thin solid line). The thick solid line shows the secular evolution of the mass and radius of the secondary star computed by Kolb \& Baraffe (in preparation). 50 stars in detached binaries with well-determined masses and radii have been plotted as open circles in the upper panel. See text for details.

$M_{2}=0.6 \mathrm{M}_{\odot}$. The main features in the upper part of the curve are the large radii at high masses (compared to the theoretical main sequence) due to thermal inequilibrium and the detachment of the secondary from its Roche lobe at $M_{2}=0.22 \mathrm{M}_{\odot}$, which is the point at which (in this model) the secondary becomes fully convective and magnetic braking is assumed to cease. During this detached phase, there is a 25 per cent fall in radius as the secondary star relaxes back to thermal equilibrium.

The lower part of the curve follows the theoretical main sequence, with the secondary star close to thermal equilibrium at low mass transfer rates, until the minimum mass for nuclear fusion is reached and the secondary becomes a degenerate, brown dwarflike object with $R_{2} \propto M_{2}^{-1 / 3}$. Note that the evolutionary sequence should be used with caution when interpreting the observed CV masses, as it represents the change in mass and radius of a single secondary star with time, whereas the observed data are a snapshot of the masses and radii of the population of secondary stars at this moment in time. To make a proper comparison with theory, one would need to compute the evolutionary tracks of a sample of secondary stars generated by a population synthesis code.

As a group, the CVs appear to lie slightly above the theoretical main sequence of Chabrier \& Baraffe (1997), but appear to fit the main sequence as defined by the distribution of detached systems very well. There are a few departures; as already noted, DX And and AE Aqr have evolved secondary stars and lie above the main sequence, while UU Aqr and IP Peg both lie above the main sequence, being undersized for their masses, but within the error bars they are still consistent with the main sequence. Rather surprisingly, those systems which we have omitted from the fitting process because of dubious $K_{\mathrm{W}}$ measurements also follow the main sequence very closely. Unfortunately, the errors on the masses and the scatter of the points in Fig. 2 do not allow us to say if the secondary stars in CVs follow the disrupted magnetic braking model, or the kinked lower main sequence of Clemens et al. (1998). ${ }^{2}$ This means our data are unable to test which of these two period gap formation mechanisms is correct (see Section 7). There is also no evidence in these data for systems which have evolved beyond the orbital period minimum and have low-mass, degenerate brown dwarf-like secondaries (see Howell, Rappaport \& Politano 1997).

Of the three short-period LMXBs, V616 Mon and QZ Vul both contain secondary stars which lie above the main sequence and are somewhat evolved, while GU Mus lies just on the main sequence (to within the error). The secondary stars in the long-period LMXBs V404 Cyg and V1033 Sco are both evolved (V1033 Sco is also very massive) and do not appear in Fig. 2.

\section{THE MASS-SPECTRAL TYPE RELATION}

An assumption often made in the estimation of system parameters in CVs is that the spectral type of the secondary star can be used to estimate its mass. In Fig. 3 we have plotted those CVs and LMXBs which have measured masses and spectral types given in Tables 1,2 and 3. Also plotted are the detached systems from Popper (1980) and Andersen (1991).

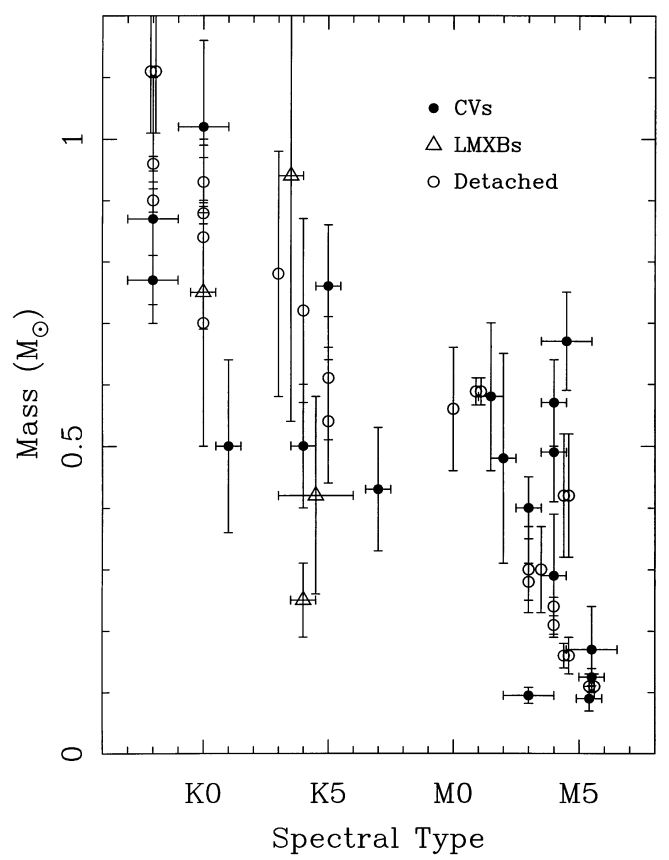

Figure 3. The masses and spectral types of the secondary stars in CVs and LMXBs. The filled circles represent the CVs, the triangles the LMXBs, and the unfilled circles the detached systems.

${ }^{2}$ This has recently been shown not to create a period gap at all, but rather two spikes at $P \sim 2 \mathrm{~h}$ and $\sim 3 \mathrm{~h}$ with the probability of discovering a $\mathrm{CV}$ in the gap no lower than discovering one outside the gap (Kolb, King \& Ritter 1998). 

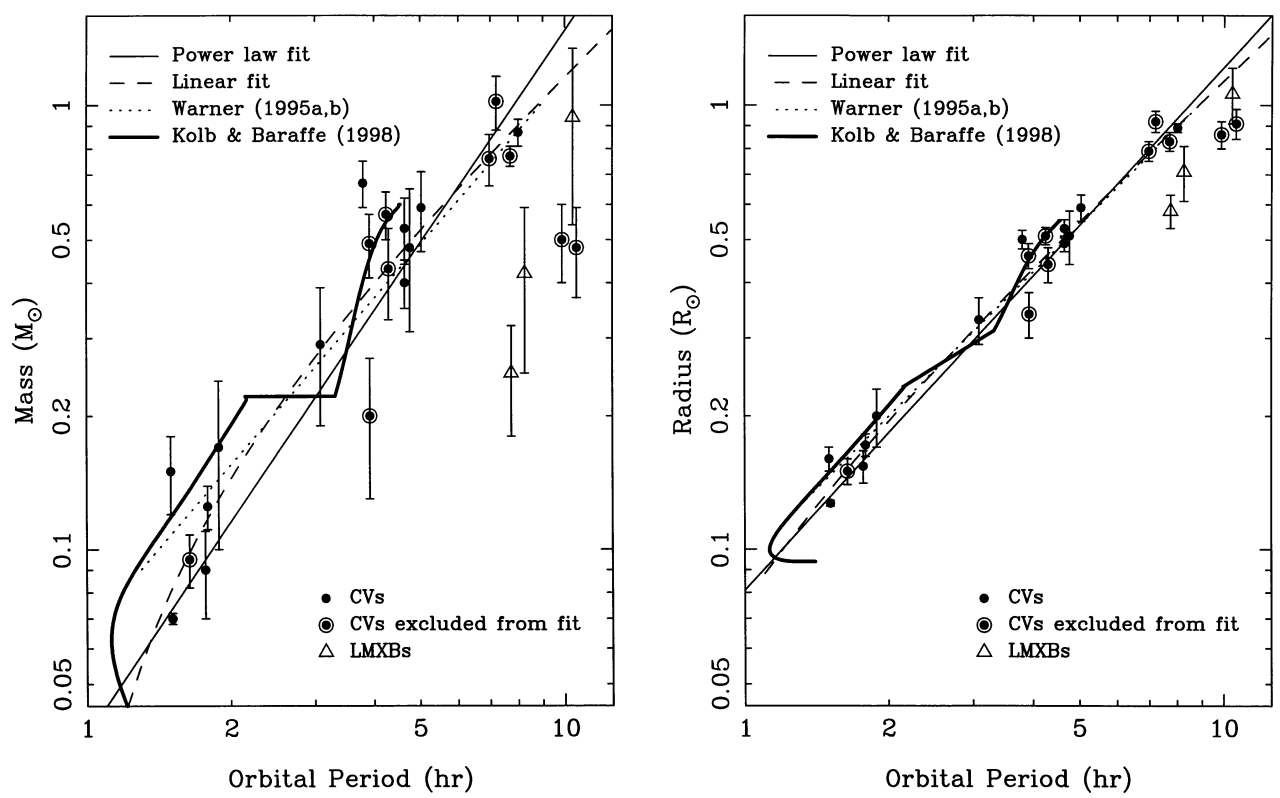

Figure 4. The masses and radii of the secondary stars in CVs versus their orbital periods. In each panel, the thin solid line is the power-law fit to the CV data (equations 8 and 11), and the dashed line is a linear fit to the CV data (equations 9 and 12). The dotted lines are the semi-empirical relations of Warner (1995a,b; equations 10 and 13). The thick solid line is the evolutionary sequence of Kolb \& Baraffe (in preparation). The ringed points have been omitted from the fits - see text for details.

The mass of the secondary is thought to be the most important factor in determining its effective temperature, and therefore spectral type (King \& Kolb, in preparation). Stehle, Ritter \& Kolb (1996) show that simple homology relations lead to a relationship between the effective temperature of the star (and therefore spectral type) and the radius of the form $T_{\text {eff }} \propto R^{\mu}$, where $|\mu| \ll 1$. Stars should therefore expand or contract without changing their spectral types by much and the spectral type of a star should be almost completely dependent on its mass.

Fig. 3 shows that this is not the case, and that there is a huge range in mass for a given spectral type. Around M5, for example, lie IP Peg (M4.5) and Z Cha (M5.5); the secondary star in IP Peg is 5 times more massive than that in $\mathrm{Z}$ Cha. Among the LMXBs there is also little or no correlation between spectral type and masses; GU Mus $(\mathrm{K} 3-4 \mathrm{~V})$ is around 4 times more massive than V616 Mon (K4V). The large scatter in masses for a given spectral type is, however, shared by the detached systems (e.g., around M4-5 and K0). So, although the secondary stars in CVs and LMXBs are not too different from main-sequence stars, there is too much variation to use the spectral types of CV and LMXB secondaries to estimate the secondary star masses, as is also the case with the detached stars.

\section{THE MASS-PERIOD AND RADIUS-PERIOD RELATIONS}

Another relation of interest is that between the orbital period and the mass of the secondary star. We have used the masses and periods listed in Table 3 to derive a mass-period relation. By fitting a power law to the data (excluding the evolved secondaries in DX And and $\mathrm{AE}$ Aqr, and the systems with dubious $K_{\mathrm{W}}$ determinations), we have derived the following relationship:

$\frac{M}{\mathrm{M}_{\odot}}=(0.038 \pm 0.003) P^{(1.58 \pm 0.09)}$.

We have also performed a linear fit and derived the following mass-period relationship:

$\frac{M}{\mathrm{M}_{\odot}}=(0.126 \pm 0.011) P-(0.11 \pm 0.04)$.

Warner (1995a,b) derived the following semi-empirical massperiod relation, which utilized his mass-radius relation (equation 7):

$\frac{M}{\mathrm{M}_{\odot}}=0.065 P^{5 / 4}$.

The mass-period relation is shown in the left-hand panel of Fig. 4. The filled circles represent the $\mathrm{CVs}$; those which are ringed have been omitted from the fit, either because they depend upon dubious $K_{\mathrm{W}}$ measurements, or because they are evolved to some degree. The thin solid line is the power-law fit (equation 8), the dashed line is the linear fit (equation 9), and the dotted line is Warner's relation (equation 10). The evolutionary sequence of Kolb \& Baraffe (in preparation) is plotted as a thick solid line.

The linear fit (unweighted rms deviation $=0.10$ ) is surprisingly better than the power-law fit ( $\mathrm{rms}=0.12)$; one would have expected a homologous relation between mass and period, and therefore a power law to be the best fit. Warner's fit is also surprisingly good ( $\mathrm{rms}=0.11$ ), but it is a poorer fit to the systems with short periods and those with the most accurate mass determinations. The evolved systems DX And and AE Aqr lie a long way from the fit, while IP Peg is twice as massive as the fits predict. The LMXBs all have lower masses than the CV fits predict. The period gap is represented in Fig. 4 by the flat Section of the evolutionary sequence at $M=0.22 \mathrm{M}_{\odot}$; the data are insufficiently accurate or numerous to say whether the secondary stars follow it. There are no stars lying on the degenerate secondary star arm of the evolutionary sequence, implying that none of the short-period systems in our sample containing the degenerate, brown dwarf-like secondaries we expect in post-period minimum CVs.

A similar procedure to that above has been followed to derive a radius-period relation for $\mathrm{CV}$ secondary stars. A power-law fit has 
been applied to the data in Table 3, again excluding DX And and AE Aqr and the systems with dubious $K_{\mathrm{W}}$ measurements. The resulting radius-period relation is

$\frac{R}{\mathrm{R}_{\odot}}=(0.081 \pm 0.019) P^{(1.18 \pm 0.04)}$.

We have also performed a linear fit, which is given by

$\frac{R}{\mathrm{R}_{\odot}}=(0.117 \pm 0.004) P-(0.041 \pm 0.018)$.

The corresponding semi-empirical radius-period relation of Warner (1995a,b) is

$\frac{R}{\mathrm{R}_{\odot}}=0.094 P^{13 / 12}$.

The right-hand panel of Fig. 4 shows the radius-period relation for CVs. The thin solid line is the power-law fit (equation 11) to the $\mathrm{CV}$ data (unringed circles), the dashed line is the linear fit (equation 12), and the dotted line is Warner's relation (equation 13). The thick solid line is the evolutionary sequence of Kolb \& Baraffe (in preparation). The rms scatters from the fits show them to be of similar quality: 0.033 for the linear fit, 0.042 for the power law, and 0.036 for Warner's relation. The correlation here is stronger than that in the mass-period diagram, as is to be expected: from equation (3), any deviation in mass from the mass-period relation must be matched by a deviation one-third the size in the radius-period diagram. The LMXBs have smaller radii than their orbital periods predict; again the deviation from the fit is around one-third of that in the mass-period plot.

\section{WHITE DWARF MASSES}

The careful selection of systems with well-measured secondary star masses and radii also provides us with the most accurately determined sample of CV white dwarf masses. In Table 4, we list the mean white dwarf mass for each of the CV subtypes, above and below the period gap, along with the standard errors, followed by the number of systems in parentheses. We have computed weighted and unweighted means for the white dwarfs - the methods used are given in the footnotes to Table 4 . The unweighted mean is probably the more reliable statistic to use, since the range of formal errors in the white dwarf mass determinations is so great; the photometric determinations which have small formal errors but certainly much larger systematic errors (e.g., OY Car) dominate in the calculation of the weighted means. The dispersions on the unweighted means are also more representative than those quoted for the weighted means. White dwarf masses are plotted against orbital period in the upper panel of Fig. 5, with each CV subtype represented by a different symbol.

The systems below the period gap have a lower mean mass than those above, which is to be expected, since high-mass white dwarfs are required to support stable conservative mass transfer from the more massive secondaries which reside in long-period CVs. Most of the white dwarf masses are consistent with $\mathrm{CO}$ white dwarfs; the white dwarfs in U Gem and IP Peg are near the minimum mass for ONeMg white dwarfs $\left(\sim 1.15 \mathrm{M}_{\odot}\right.$; e.g. Iben, Ritossa \& GarciaBerro 1997), but none are light enough to be He white dwarfs $\left(\lessgtr 0.4 \mathrm{M}_{\odot}\right)$. The mean mass for systems below the gap is probably

Table 4. The mean white dwarf masses for each of the CV subtypes, for systems below the period gap, above the period gap, and in total. The standard errors are also listed, with the numbers of systems used in determining the mean given in parentheses. Note that DQ Her has been counted as both a classical nova and an intermediate polar.

\begin{tabular}{|c|c|c|c|}
\hline \multicolumn{4}{|c|}{ A. Unweighted Average ${ }^{a}$} \\
\hline Sample & Below period gap & Above period gap & All periods \\
\hline Dwarf novae & $0.73 \pm 0.10$ & $0.84 \pm 0.29(7)$ & $0.80 \pm 0.24$ \\
\hline Nova-likes & - & $0.79 \pm 0.08$ & $0.79 \pm 0.08$ \\
\hline Polars & $0.76^{*}$ & $0.69 \pm 0.35(2)$ & $0.71 \pm 0.25$ \\
\hline Intermediate polars & $0.49^{*}$ & $0.70 \pm 0.13(2)$ & $0.63 \pm 0.15$ \\
\hline Classical novae & - & $0.82 \pm 0.31(2)$ & $0.82 \pm 0.31(2)$ \\
\hline All systems & $0.69 \pm 0.13(6)$ & $0.80 \pm 0.22(16)$ & $0.77 \pm 0.21$ \\
\hline \multicolumn{4}{|c|}{ B. Weighted Average ${ }^{b}$} \\
\hline Sample & Below period gap & Above period gap & All periods \\
\hline Dwarf novae & $0.68 \pm 0.01(4)$ & $0.75 \pm 0.03(7)$ & $0.69 \pm 0.01$ \\
\hline Nova-likes & - & $0.82 \pm 0.06$ & $0.82 \pm 0.06$ \\
\hline Polars & $0.76 \pm 0.30$ & $0.58 \pm 0.09(2)$ & $0.60 \pm 0.09$ \\
\hline Intermediate polars & $0.49 \pm 0.03$ & $0.63 \pm 0.06(2)$ & $0.52 \pm 0.03$ \\
\hline Classical novae & - & $0.85 \pm 0.05(2)$ & $0.85 \pm 0.05$ \\
\hline All systems & $0.66 \pm 0.01(6)$ & $0.78 \pm 0.02(16)$ & $0.68 \pm 0.01(22)$ \\
\hline $\begin{array}{l}\text { Webbink (1990) } \\
\text { All systems }\end{array}$ & $0.66 \pm 0.01(26)$ & $0.81 \pm 0.04(58)$ & $0.74 \pm 0.04(84)$ \\
\hline
\end{tabular}

*No associated error.

$$
\begin{aligned}
{ }^{a} \bar{M} & =\frac{1}{N} \sum_{i=1}^{N} M_{i} & \sigma_{M}^{2} & =\frac{1}{N-1} \sum_{i=1}^{N}\left(M_{i}-\bar{M}\right)^{2} \\
{ }^{b} \bar{M} & =\sum_{i=1}^{N} \frac{M_{i}}{\sigma_{i}^{2}} / \sum_{i=1}^{N} \frac{1}{\sigma_{i}^{2}} & \sigma_{M}^{2} & =\left(\sum_{i=1}^{N} \frac{1}{\sigma_{i}^{2}}\right)^{-1}
\end{aligned}
$$




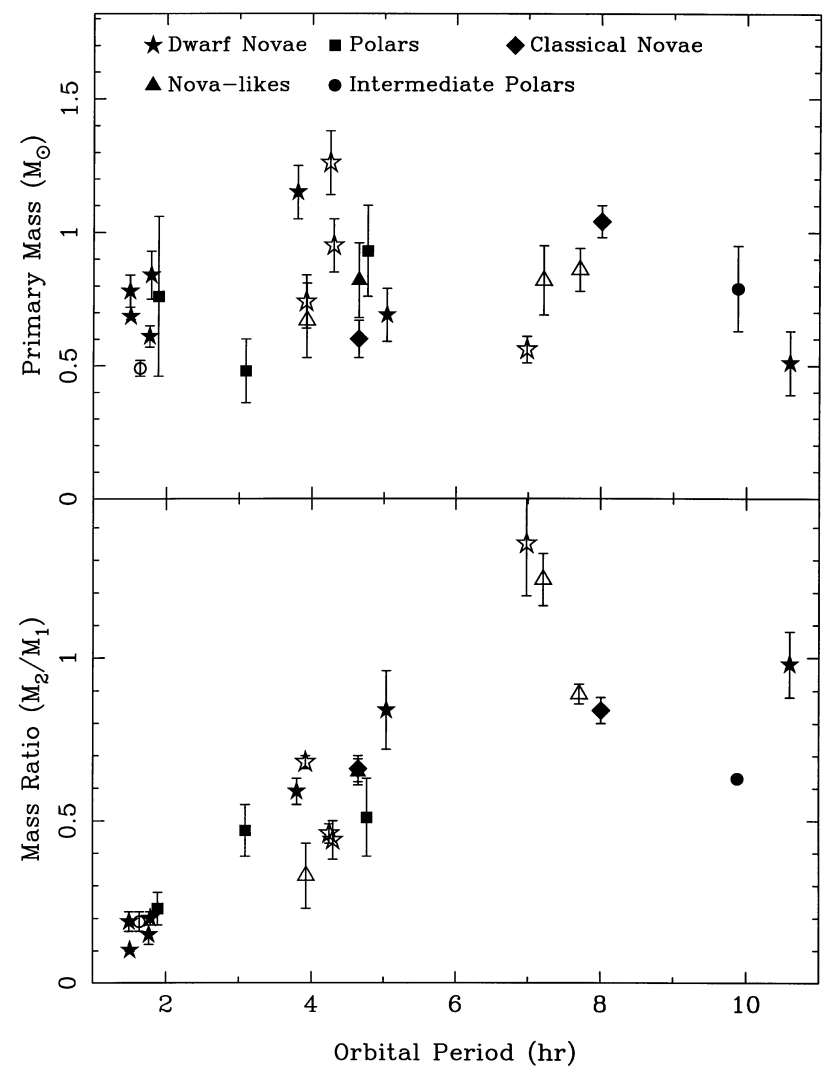

Figure 5. The masses of the white dwarfs and the mass ratios of CVs plotted against orbital period. Open symbols are used for those mass determinations which used unreliable $K_{\mathrm{W}}$ measurements.

more representative of the class as a whole, since strong selection effects come into play: it is thought that up to 99 per cent of the actual CV population are below the gap (Kolb 1993 - but see also Patterson, in preparation), despite the fact that they comprise only about 40 per cent of those observed. The white dwarf masses show no trends with CV subtype.

Webbink (1990) performed statistics on a larger sample of white dwarf masses, but a large number of these determinations were unreliable, depending on measurements of emission-line profiles (FWHM, double-peak separation, rms linewidths) which had been calibrated against a few well observed double-lined CVs and Algols. It is, however, worth comparing Webbink's figures with ours, as his mean white dwarf masses are the most often quoted. The mean masses derived by Webbink for all systems, and for those above and below the period gap, are listed in Table 4 . We reach the same general conclusions as Webbink, namely that the mean white dwarf mass above the period gap is higher than that below, and that the overall mean mass is $0.76 \pm 0.22 \mathrm{M}_{\odot}$, higher than the mean mass of single white dwarfs $\left(0.6 \mathrm{M}_{\odot}\right.$, e.g; Bergeron, Liebert \& Fulbright 1995).

The mass ratio-orbital period diagram in the lower panel of Fig. 5 shows the near-linear relation we expect, since the secondary stars' masses are well correlated with their orbital periods. The scatter in this is largely due to the scatter in white dwarf masses. Again there is no trend according to subtype; the two systems with anomalously large mass ratios, EM Cyg and $\mathrm{AC} \mathrm{Cnc}$, are a dwarf nova and a nova-like respectively. Note that this sample of accurate mass ratio determinations is not exhaustive; there exist several systems which have the mass ratio determined, but which do not have accurate mass determinations (e.g., because the inclination is unknown.)

In our sample, the unweighted mean value of the mass ratio (performed on those systems which do not have dubious $K_{\mathrm{W}}$ values) is $\bar{q}=0.17 \pm 0.05$ below the period gap and $0.70 \pm 0.15$ above the period gap. These values are consistent with the statistical analysis of eclipse durations by Bailey (1990), who found that the mean value of the mass ratio should be $\bar{q}=0.13 \pm 0.03$ below the gap and $0.65 \pm 0.12$ above the gap.

\section{DISCUSSION}

\subsection{Are CV secondaries main-sequence stars?}

The spectral type-period diagram (Fig. 1) shows that the secondary stars in CVs lie very close to the main sequence defined by the detached systems. There is little differerence between the distribution of CV secondaries and that of the detached stars, up to an orbital period of about $7-8 \mathrm{~h}$. Beyond that, for the reasons stated in Section 1, the secondary stars are required to be evolved, and we see AE Aqr $(P=9.88 \mathrm{~h})$ and DX And $(P=10.60 \mathrm{~h})$ lying well above the rest of the distribution with later spectral type than the fit predicts.

The mass-radius diagram (Fig. 2) clearly shows that the secondary stars in CVs are on the whole indistinguishable from the observed main-sequence stars in detached binary systems. Again, the only outlying points are AE Aqr and DX And, which almost certainly have evolved secondary stars. The most interesting question from a mass-determination point of view, however, is not whether secondary stars as a whole are main-sequence stars, but rather at what point can we no longer apply a main-sequence massradius relationship. From Figs 1 and 2 we can say that CVs with periods of up to $7-8 \mathrm{~h}$ almost always have main-sequence secondary stars, although one must always beware of the existence of systems with peculiar secondaries and use the mass-radius relation with caution (e.g., by correctly propagating the errors in our massradius relation when determining masses).

We have seen that the expected divergence from the main sequence does occur in long-period CVs, but there is as yet no firm evidence for post-period minimum $\mathrm{CV}$ s with degenerate brown dwarf-like secondaries in short-period CVs. The evolutionary sequence of Kolb \& Baraffe (in preparation) shows us where to look, but as yet there have been no brown dwarf-like secondaries detected in CVs either by their spectral types (e.g., by looking for spectral types much later than predicted by equation 4 ) or by their position on the mass-radius diagram (but see Howell, Hauschildt \& Dhillon 1998). This does not mean, of course, that there are no postperiod minimum CVs; these systems should be intrinsically very faint, and near the period minimum (where WZ Sge and many other suspected post-period minimum CVs lie) their secondary stars will be difficult to distinguish from late-type main-sequence stars.

\subsection{Are LMXB secondaries main-sequence stars?}

The spectral types of the LMXBs seem to follow the same distribution as the CVs with orbital period, while their radii are larger than main-sequence stars of the same type, suggesting they are either somewhat evolved (as predicted by King \& Kolb 1997), or else have expanded towards a new state of thermal equilibrium due to the level of X-ray irradiation to which they are exposed (Podsiadlowski 1991). The upper orbital period limit above which secondary stars cannot be on the empirical main sequence rises 
(from around $9 \mathrm{~h}$ for $\mathrm{CVs}$ ) to around $15 \mathrm{~h}$, using the theoretical upper neutron star mass limit of $\sim 3 \mathrm{M}_{\odot}$ with a typical A0 mainsequence star of mass $2.40 \mathrm{M}_{\odot}$ and radius $1.87 \mathrm{R}_{\odot}$ (Gray 1992). In LMXB black hole candidates, of course, there is no such limit to the mass of the black hole and therefore no limit on the secondary star.

\subsection{Consequences for the disrupted magnetic braking model}

The generally accepted mechanism for the formation of the period gap in CVs is the disrupted magnetic braking model. Above the period gap, magnetic braking is the dominant mechanism for the angular momentum loss which drives mass transfer. As the $\mathrm{CV}$ moves towards a period of $3 \mathrm{~h}$, the secondary star's mass falls to around $0.25 \mathrm{M}_{\odot}$, at which point it becomes (almost) fully convective (Kolb 1993). Magnetic braking is then thought to cease, allowing the secondary, which due to its high mass transfer rate has been out of thermal equilibrium, to relax and shrink inside its Roche lobe. This cuts off mass transfer (and the cataclysmic behaviour) until further loss of angular momentum via gravitational radiation reduces the period to around $2 \mathrm{~h}$, at which point the secondary comes back into contact with its Roche lobe and recommences mass transfer (Rappaport, Joss \& Verbunt 1983; Spruit \& Ritter 1983).

A mass-radius diagram would be expected to show a 'kink' at around a mass of $0.25 \mathrm{M}_{\odot}$, as the $\mathrm{CV}$ secondary stars revert from being out of thermal equilibrium and therefore undermassive for their radii above the period gap, to being thermally relaxed at low masses below the period gap. This is shown in the secular evolution model plotted in the lower panel of Fig. 2. If a star enters the period gap at $P_{\text {above }}=3.0 \mathrm{hr}$ and emerges at $P_{\text {below }}=2.0 \mathrm{hr}$, without having lost any further mass, then, using equation (3), its radius must have shrunk by a factor

$\left(\frac{R_{\text {below }}}{R_{\text {above }}}\right)=\left(\frac{P_{\text {below }}}{P_{\text {above }}}\right)^{2 / 3}=0.763$,

i.e., a decrease of almost 25 per cent. A narrower period gap, e.g., $2.2-2.8 \mathrm{~h}$, would see a smaller drop in the radius at the top of the period gap, in this case 15 per cent.

We are unable to see the expected sudden drop in Fig. 2, although the data are sparse in the crucial region around $0.25 \mathrm{M}_{\odot}$, the only system there being AM Her. In this interesting region, just above the period gap, there is a dearth of dwarf novae; instead, the $\mathrm{CV}$ population is dominated by peculiar nova-likes such as the SW Sex stars (Dhillon 1996), which have intrinsically bright discs and almost undetectable secondary stars, and for which determination of system parameters is therefore very difficult.

Clemens et al. (1998) see a dip in the colour-magnitude diagram for $\mathrm{M}$ dwarfs at $M_{V} \sim 12$ (around $0.25 \mathrm{M}_{\odot}$ ). They suggest that this means that the radius of the secondary star in a CV would shrink more rapidly in this region for the same rate of mass-loss, leading to the rapid crossing of the $2-3 \mathrm{~h}$ orbital period range. This would then be a possible mechanism for the formation of the period gap. This interpretation has been disputed by Kolb et al. (1998), who show that if the mass-radius relation of Clemens et al. held, then, rather than producing a period gap, the period distribution would have two spikes at the upper and lower edges of the 'gap', with the probability of discovering CVs inside the 'gap' the same as discovering them outside the 'gap.'

Unfortunately, we are not yet at the stage where we can confirm or disprove the disrupted magnetic braking model observationally. Our data set is simply too sparse and insufficiently accurate. Several more accurate measurements of secondary star masses and radii are needed, especially around the period gap (in which there are sadly few secondary star detections) in order to differentiate between these competing period-gap formation theories.

\section{CONCLUSIONS}

We draw the following conclusions.

(i) We find that there are a total of 55 reliable spectral type determinations and only 14 reliable mass determinations of $\mathrm{CV}$ secondary stars (10 and 5, respectively, in the case of LMXBs).

(ii) We have derived new mass-radius, mass-period, radiusperiod and spectral type-period relations for CV secondary stars, using a carefully selected sample of CVs with well-measured system parameters.

(iii) The secondary stars in CVs with periods below 7-8 h are, as a group, indistinguishable from main-sequence stars in detached systems in terms of spectral type, mass and radius.

(iv) The secondary stars in LMXBs show some evidence for evolution, with radii which are slightly too large for their masses.

(v) We have shown that the assumption that the spectral type of the secondary star in CVs and LMXBs provides a good estimate of its mass is not a good one.

(vi) We have calculated the mean white dwarf mass in CVs, for the various $\mathrm{CV}$ subtypes, both above the period gap (where we find $\bar{M}_{1}=0.80 \pm 0.22 \mathrm{M}_{\odot}$ ) and below the period gap (where we find $\left.\bar{M}_{1}=0.69 \pm 0.13 \mathrm{M}_{\odot}\right)$.

(vii) We have shown that accurate measurements of masses and radii in $\mathrm{CV}$ secondary stars can be used to constrain $\mathrm{CV}$ evolution and provide evidence for or against the disrupted magnetic braking theory.

(viii) We have demonstrated the need for many more accurate $\mathrm{CV}$ mass measurements, especially around the period gap, to test the disrupted magnetic braking model, and among the faint shortperiod $\mathrm{CVs}$ to search for the predicted post-period minimum systems.

\section{ACKNOWLEDGMENTS}

We are indebted to Mike Politano and Uli Kolb for providing us with secular evolution models and Ron Webbink for supplying his data set of CV system parameters. We also thank Uli Kolb and Mike Politano for invaluable discussions on secondary stars and the evolution of CVs, and the referee, Hans Ritter, for his careful reading of the manuscript and suggestions for improvements. DAS is supported by a PPARC studentship.

\section{REFERENCES}

Allen C. W., 1976, Astrophysical Quantities. Athlone Press, London Andersen J., 1991, A\&AR, 3, 91

Andronov I. L., Fuhrmann B., 1987, Inf. Bull. Variable Stars No. 2976

Bailey J., 1990, MNRAS, 243, 57

Baptista R., Steiner J. E., Cieslinski D., 1994, ApJ, 433, 332

Baptista R., Horne K., Hilditch R. W., Mason K. O., Drew J. E., 1995, ApJ, 448, 395

Baptista R., Catalán, M. S., Horne K., Zilli D., 1998, MNRAS, in press, (astro-ph9806157)

Barrera L. H., Vogt N., 1989, A\&A, 220, 99

Barwig H., Ritter H., Baernbantner O., 1994, A\&A, 288, 204

Beekman G., Shahbaz T., Naylor T., Charles P. A., Wagner R. M., Martini P., 1997, MNRAS, 290, 303

Bergeron P., Liebert J., Fulbright M. S., 1995, ApJ, 444, 810

Berriman G., Szkody P., Capps R. W., 1985, MNRAS, 217, 327

Beuermann K., Pakull M. W., 1984, A\&A, 136, 250 
Beuermann K., Thomas H. C., 1990, A\&A, 230, 326

Beuermann K., Thomas H. C., Giommi P., Tagliaferri G., Schwope A. D., 1989, A\&A, 219, L7

Billington I., Marsh T. R., Dhillon V. S., 1996, MNRAS, 278, 673

Bruch A., 1987, A\&A, 172, 187

Bruch A., Vrielmann S., Hessman F. V., Kochsiek A., Schimpke T., 1997, A\&A, 327, 1107

Buckley D. A. H., Shafter A. W., 1995, MNRAS, 275, L61

Casares J., Charles P. A., 1994, MNRAS, 271, L5

Casares J., Martin A. C., Charles P. A., Martin E. L., Rebolo R., Harlaftis E. T., Castro-Tirado A. J., 1995, MNRAS, 276, L39

Casares J., Mouchet M., Martinez-Pais I. G., Harlaftis E. T., 1996, MNRAS, 282,182

Casares J., Martin E. L., Charles P. A., Molaro P., Rebolo R., 1997, New Astron., 1, 299

Chabrier G., Baraffe I., 1997, A\&A, 327, 1039

Chevalier C., Ilovaisky S. A., 1996, A\&A, 312, 105

Clemens J. C., Reid I. N., Gizis J. E., O’Brien M., 1998, ApJ, 496, 352

Crampton D., Cowley A. P., Fisher W. A., 1986, ApJ, 300, 788

Cropper M. S., 1986, MNRAS, 222, 853

Davey S. C., Smith R. C., 1996, MNRAS, 280, 481

Dhillon V. S., 1996, in Evans A., Wood J. H., eds, Cataclysmic Variables and Related Objects. Kluwer Academic Publishers, Netherlands, p. 3

Dhillon V. S., Marsh T. R., 1995, MNRAS, 275, 89

Dhillon V. S., Marsh T. R., Duck S. R., Rosen S. R., 1997, MNRAS, 285, 95

Drew J. E., Jones D. H. P., Woods J. A., 1993, MNRAS, 260, 803

Echevarría J., 1983, Rev. Mex. Astron. Astrofis., 8, 109

Eggleton P., 1983, ApJ, 268, 368

Eracleous M., Horne K., Robinson E. L., Zhang E.-H., Marsh T. R., Wood J. H., 1994, ApJ, 433, 313

Fiedler H., Barwig H., Mantel K. H., 1997, A\&A, 327, 173

Fiedler S. H., 1994, Diploma thesis. LMU Munich

Frank J., King A. R., Raine D. J., 1992, Accretion Power in Astrophysics, 2nd edition. Cambridge Univ. Press, Cambridge

Friend M. T., Martin J. S., Smith R. C., Jones D. H. P., 1990a, MNRAS, 246, 637

Friend M. T., Martin J. S., Smith R. C., Jones D. H. P., 1990b, MNRAS, 246, 654

Glenn J., Howell S. B., Schmidt G. D., Liebert J., Grauer A. D., Wagner R. M., 1994, ApJ, 424, 967

Goetz W., 1986, Inf. Bull. Variable Stars, 2918

Gray D. F., 1992, The Observation and Analysis of Stellar Photospheres. Cambridge Univ. Press, Cambridge

Hamada T., Salpeter E. E., 1961, ApJ, 134, 683

Harlaftis E., Horne K., Filippenko A. V., 1996, PASP, 108, 762

Harlaftis E. T., Steeghs D., Horne K., Filippenko A. V., 1997, AJ, 114, 1170

Harrop-Allin M. K., Warner B., 1996, MNRAS, 279, 219

Haswell C. A., Robinson E. L., Horne K., Stiening R. F., Abbott T. M. C., 1993, ApJ, 411, 802

Haswell C. A., Patterson J., Thorstensen J. R., Hellier C., Skillman D. R., 1997, ApJ, 476, 847

Hawkins N. A., Smith R. C., Jones D. H. P., 1990, in Mauche C. W., ed., Accretion Powered Compact Binaries. Cambridge Univ. Press, Cambridge, p. 113

Hellier C., 1996, in Evans A., Wood J. H., eds, Cataclysmic Variables and Related Objects. Kluwer Academic Publishers, Netherlands, p. 143

Hellier C., Mason K. O., Rosen S. R., Cordova F. A., 1987, MNRAS, 228 , 463

Hessman F. V., 1988, A\&AS, 72, 515

Hilditch R., 1995, MNRAS, 273, 675

Horne K., Verbunt F., Schneider D. P., 1986a, MNRAS, 218, 63

Horne K., Wade R. A., Szkody P., 1986b, ApJ, 219, 791

Horne K., Wood J. H., Steining R. F., 1991, ApJ, 378, 271

Horne K., Welsh W. F., Wade R. A., 1993, ApJ, 410, 357

Howell S. B., Mason K. O., Reichert G. A., Warnock W., 1988, MNRAS, 233,79
Howell S. B., Szkody P., Kreidl T. J., Mason K. O., Puchnarewicz E. M., 1990, PASP, 102, 758

Howell S. B., Liebert J., Mason K. O., 1994, Inf. Bull. Variable Stars No. 4072

Howell S. B., Rappaport S. A., Politano M., 1997, MNRAS, 287, 929

Howell S. B., Hauschildt P., Dhillon V. S., 1998, ApJ, 494, 223

Iben I., Ritossa C., Garcia-Berro E., 1997, ApJ, 489, 772

Jaschek C., Jaschek M., 1987, The Classification of Stars. Cambridge Univ. Press, Cambridge

Johnston H. M., Kulkarni S. R., 1992, ApJ, 396, 267

Jurcevic J. S., Honeycutt R. K., Schlegel E. M., Webbink R. F., 1994, PASP, 106, 481

Kaitchuck R. H., 1989, PASP, 101, 1129

King A. R., Kolb U., 1997, ApJ, 481, 918

Kolb U., 1993, A\&A, 271, 149

Kolb U., King A. R., Ritter H., 1998, MNRAS, 298, L29

Kraft R., 1964, ApJ, 139, 457

Kuerster M., Barwig H., 1988, A\&A, 199, 201

Mantel K. H., Barwig H., Haefner R., Schoembs R., 1987, Ap\&SS, 131, 501

Marsh T. R., 1988, MNRAS, 231, 1117

Marsh T. R., 1990, ApJ, 357, 621

Marsh T. R., Robinson E. L., Wood J. H., 1994, MNRAS, 266, 137

Martin J. S., Jones D. H. P., Smith R. C., 1987, MNRAS, 224, 1031

Martin J. S., Jones D. H. P., Friend M. T., Smith R. C., 1989, MNRAS, 240, 519

Mateo M., Szkody P., Garnavich P. M., 1991, ApJ, 370, 370

McClintock J. E., Remillard R. A., 1986, ApJ, 308, 110

McClintock J. E., Remillard R. A., 1990, ApJ, 350, 386

Mukai K., Charles P. A., 1986, MNRAS, 222, L1

Mukai T. et al., 1990, MNRAS, 245, 385

Nauenberg M., 1972, ApJ, 175, 417

Okazaki A., Kitamura M., Yamasaki A., 1982, PASP, 94, 162

Orosz J. A., Bailyn C. D., McClintock J. E., Remillard R. A., 1996, ApJ, 468, 380

Orosz J. A., Bailyn C. D., 1997, ApJ, 477, 876

Paczynski B., 1971, ARA\&A, 9, 183

Patterson J., 1984, ApJS, 54, 443

Podsiadlowski P., 1991, Nat, 350, 136

Popper D., 1980, ARA\&A, 18, 115

Puchnarewicz E. M., Mason K. O., Murdin P. G., Wickramasinghe D. T., 1990, MNRAS, 244, L20

Rappaport S. A., Joss P. C., Verbunt F., 1983, ApJ, 275, 713

Reid I. N., Gizis J. E., 1997, AJ, 114, 1992

Reinsch K., 1994, A\&A, 281, 108

Remillard R. A., Schachter J. F., Silber A. D., 1994, ApJ, 426, 288

Ringwald F. A., Thorstensen J. R., Hamwey R. M., 1994, MNRAS, 271, 323

Ringwald F. A., Thorstensen J. R., Honeycutt R. K., Smith R. C., 1996, AJ, 111,2077

Ritter H., 1983, in Livio M., Shaviv G., eds., Proc. IAU Colloq. 72, Cataclysmic Variables and Related Objects. Reidel, Dordrecht, p. 257

Ritter H., Kolb U., 1998, A\&AS, 129, 83

Robinson E. L. et al., 1995, ApJ, 443, 295

Rutten R. G. M., Dhillon V. S., 1994, A\&A, 288, 773

Rutten R. G. M., Dhillon V. S., 1996, in Evans A., Wood J. H., eds, Cataclysmic Variables and Related Objects. Kluwer Academic Publishers, Netherlands, p. 21

Rutten R. G. M., Dhillon V. S., Horne K., Kuulkers E., 1994, A\&A, 283, 441 Sarna M. J., Pych W., Smith R. C., 1995, Inf. Bull. Variable Stars No. 4165 Schaefer B. E., Ringwald F. A., 1995, ApJ, 447, L45

Schlegel E. M., Kaitchuck R. H., Honeycutt R. K., 1984, ApJ, 280, 235

Schlegel E. M., Honeycutt R. K., Kaitchuck R. H., 1986, ApJ, 307, 760

Schneider D. P., Young P., Schectman S. A., 1981, ApJ, 245, 644

Schwope A. D., Naundorf C. E., Thomas H. C., Beuermann K., 1991, A\&A, 224,373

Schwope A. D., Thomas H. C., Beuermann K., Reinsch K., 1993, A\&A, 267, 103

Schwope A. D., Thomas H. C., Beuermann K., Burwitz V., Jordan S., Haefner R., 1995, A\&A, 293, 764 
Sekiguchi K., 1992, Nat, 358, 563

Shafter A. W., 1983, Inf. Bull. Variable Stars No. 2377

Shafter A. W., Harkness R. P., 1986, AJ, 92, 658

Shafter A. W., Szkody P., Thorstensen J. R., 1986, ApJ, 308, 765

Shafter A. W., Reinsch K., Beuermann K., Misselt K. A., Buckley D. A. H., Burwitz V., Schwope A. D., 1995, ApJ, 443, 319

Shahbaz T., 1998, MNRAS, 298, 153

Shahbaz T., Wood J. H., 1996, MNRAS, 282, 362

Shahbaz T., Naylor T., Charles P. A., 1994a, MNRAS, 268, 756

Shahbaz T., Ringwald F. A., Bunn J. C., Naylor T., Charles P. A., Casares J., 1994b, MNRAS, 271, L10

Shahbaz T., Bandyopadhyay R., Charles P. A., Naylor T., 1996a, MNRAS, 282, 977

Shahbaz T., Van Der Hooft F., Charles P. A., Casares J., Van Paradijs J., 1996b, MNRAS, 282, L47

Shahbaz T., Naylor T., Charles P. A., 1996c, MNRAS, 282, 1437

Silber A., Remillard R. A., Horne K., Bradt H. V., 1994, ApJ, 424, 955

Skillman D. R., Patterson J., Thorstensen J. R., 1995, PASP, 107, 545

Smak J., 1976, Acta Astron., 26, 277

Smak J., 1993, Acta Astron., 43, 121

Smith D. A., Dhillon V. S., Marsh T. R., 1998, MNRAS, 296, 465

Smith R. C., 1995, in Buckley D. A. H., Warner B., eds, ASP Conf. Ser. Vol. 85, Cape Workshop on Magnetic Cataclysmic Variables. Astron. Soc. Pac., San Francisco p. 417

Smith R. C., Cameron A. C., Tucknott D. S., 1993, in Regev O., Shaviv G., eds, Cataclysmic Variables and Related Physics. Inst. Phys. Publ., Bristol, p. 70

Smith R. C., Sarna M. J., Catalan M. S., Jones D. H. P., 1997, MNRAS, 287, 271

Southwell K. A., Still M. D., Smith R. C., Martin J. S., 1995, A\&A, 302, 90

Spruit H. C., Ritter H., 1983, A\&A, 124, 267

Stehle R., Ritter H., Kolb U., 1996, MNRAS, 279, 581

Sterken C., Vogt N., Freeth R., Kennedy H. D., Page A. A., Marino B. F., Walker W. S. G., 1983, A\&A, 118, 325

Still M. D., 1996, in Evans A., Wood J. H., eds, Cataclysmic Variables and Related Objects. Kluwer Academic Publishers, Netherlands, p. 25

Stobie R. S., Okeke P. N., Buckley D. A. H., O’Donoghue D., 1996, MNRAS, 283, L127

Stover R. J., 1981a, ApJ, 249, 684

Stover R. J., 1981b, ApJ, 249, 693

Stover R. J., Robinson E. L., Nather R. E., 1981, ApJ, 248, 696

Szkody P., Wade R. A., 1981, ApJ, 251, 201

Szkody P., Williams R. E., Margon B., Howell S. B., Mateo M., 1992, ApJ, 387,357

Thorstensen J. R., Ringwald F. A., 1995, Inf. Bull. Variable Stars No. 4249

Thorstensen J. R., Ringwald F. A., 1997, PASP, 109, 483

Thorstensen J. R., 1997, PASP, 109, 1241

Tovmassian G. H., Greiner J., Zickgraf F. J., Kroll P., Krautter J., Thiering I., Zharykov S. V., Serrano A., 1997, A\&A, 328, 571

Wade R. A., Horne K., 1988, ApJ, 324, 411

Warner B., 1995a, Cataclysmic Variable Stars. Cambridge Univ. Press, Cambridge

Warner B., 1995b, Ap\&SS, 232, 89

Watson M. G. et al., 1995, MNRAS, 273, 681

Webbink R. F., 1990, in Mauche C. W., ed., Accretion Powered Compact Binaries. Cambridge Univ. Press, Cambridge, p. 177

Welsh W. F., Horne K., Gomer R., 1993, ApJ, 410, L39

Wickramasinghe D. A., Bailey J., Meggitt S. M. A., Ferrario L., Hough J., Tuohy I. R., 1991, MNRAS, 251, 28

Wolf S., Mantel K. H., Horne K., Barwig H., Schoembs R., Baernbantner O., 1993, A\&A, 273, 160

Wood J., Horne K., Berriman G., Wade R., O’Donoghue D., Warner B., 1986, MNRAS, 219, 629

Wood J. H., Horne K., Berriman G., Wade R. A., 1989, ApJ, 341, 974

Young P., Schneider D. P., 1981, ApJ, 247, 960

Young P., Schneider D. P., Schectman S. A., 1981, ApJ, 245, 1043

Zhang E., Robinson E. L., Stiening R. F., Horne K., 1995, ApJ, 454, 447

\section{APPENDIX A: NOTES ON INDIVIDUAL SYSTEMS}

(i) V2051 Oph. Baptista et al. (1998) used a purely photometric method to find the masses of the components in this dwarf nova. The white dwarf and bright spot eclipses are both clearly visible in the light curve, so the additional measurement of $\Delta \phi_{1 / 2}$ allowed the system parameters to be determined using the white dwarf massradius relation of Hamada \& Salpeter (1961) and a Monte Carlo simulation.

(ii) OY Car. Wood et al. (1989) also used the photometric method to determine the masses in this dwarf nova. Several other mass determinations cited by Wood et al. (1989) all give higher values for $M_{2}$.

(iii) EX Hya. This intermediate polar has a detectable secondary star. Hellier (1996) combined $K_{\mathrm{W}}$ (Hellier et al. 1987) and $K_{\mathrm{R}}$ (measured using skew mapping; Smith, Cameron \& Tucknott 1993) with $\Delta \phi_{1 / 2}$ to obtain the mass. We have calculated the radius of the secondary star using equation (3).

(iv) HT Cas. Horne, Wood \& Steining (1991) performed a similar analysis to that of Wood et al. (1989).

(v) Z Cha. Wood et al. (1986) used the same technique as that applied to V2051 Oph, and obtained values of $0.081 \pm 0.003 \mathrm{M}_{\odot}$ and $0.149 \pm 0.002 \mathrm{R}_{\odot}$ for the mass and radius of the secondary star respectively. Wade \& Horne (1988) derived another mass estimate, using the mass ratio and inclination $i$ derived by Wood et al. (1986), but rather than using the white dwarf ingress/egress timings, they obtained a measurement of $K_{\mathrm{R}}$. The higher values derived by Wade $\&$ Horne (1988) are those used throughout this paper. The inconsistency between the two techniques cannot be satisfactorily explained by errors in the Hamada-Salpeter relation or the presence of a thick boundary layer above the surface of the white dwarf.

(vi) ST LMi. Shahbaz \& Wood (1996) measured the secondary star's radial velocity in this non-eclipsing polar, and determined the masses using the value of $i$ derived from polarimetric measurements (Cropper 1986).

(vii) AM Her. We have calculated the mass and radius of the secondary in the prototypical polar, using the values of $K_{\mathrm{R}}$ and $V \sin i$ determined by Southwell et al., with the appropriate $k$ correction given by Davey \& Smith (1996), and $i$ determined using polarimetry by Wickramasinghe et al. (1991). Southwell et al. found an 8 per cent bias in their measurement of $V \sin i$; we therefore adopt $V \sin i=100 \pm 10 \mathrm{~km} \mathrm{~s}^{-1}$. Note that Shahbaz \& Wood (1996) found $V \sin i=68 \pm 12 \mathrm{~km} \mathrm{~s}^{-1}$, using fewer spectra, and did not correct for irradiation effects. We therefore omit this measurement from the calculation. See Appendix C.

(viii) IP Peg. Marsh (1988) applied the light centres method to obtain $K_{\mathrm{W}}$. Martin et al. (1989) found $K_{\mathrm{R}}$ and $\Delta \phi_{1 / 2}$ which provide a direct spectroscopic determination of the mass and radius of the secondary star.

(ix) $C N$ Ori. Friend et al. (1990a) measured $K_{\mathrm{R}}$, and combined this with possibly unreliable values of $i$ and $K_{\mathrm{W}}$ derived from a radial velocity study by Mantel et al. (1987), the details of which are unpublished.

(x) UU Aqr. Baptista, Steiner \& Cieslinski (1994) used the method of Wood et al. (1989) to calculate the system parameters of this nova-like from photometry of the eclipses. Unfortunately, the eclipse light curves do not have the shape usually required for the eclipse technique to be completely valid. We therefore group this mass determination with the possibly unreliable $K_{\mathrm{W}}$ determinations. 
(xi) U Gem. The value of $K_{\mathrm{R}}$ found by Friend et al. (1990a) was combined with $K_{\mathrm{W}}$ measured by Stover (1981a) and $i$ estimated by Smak (1976) from the location of the bright spot to calculate the masses.

(xii) BD Pav. $K_{\mathrm{R}}$ and $V \sin i$ were measured by Friend et al. (1990a), and have been combined with the measurement of $\Delta \phi_{1 / 2}$ (Harrop-Allin \& Warner 1996, and references therein) to obtain the mass and radius of the secondary star. See Appendix C.

(xiii) $D Q$ Her. Horne, Welsh \& Wade (1993) measured $K_{\mathrm{R}}$ and $V \sin i$, and combined these with previous measurements of $K_{\mathrm{W}}$ and $\Delta \phi_{1 / 2}$ to obtain a full set of system parameters, using a Monte Carlo simulation.

(xiv) IX Vel. Beuermann \& Thomas (1990) detected emission lines emanating from the secondary star in this bright, non-eclipsing nova-like, which effectively make it a double-lined binary. Using kinematic and geometric modelling, and using the Balmer line light curves to constrain $i$, they obtained the mass and radius of the secondary star.

(xv) V895 Cen (= EUVE J1429 + 38.0). The mass and radius of the secondary have recently been determined by Buckley et al. (in preparation). $K_{\mathrm{R}}$ and $V \sin i$ were obtained using the NaI 8190-A absorption doublet, and $i$ was found from ellipsoidal variations.

(xvi) EX Dra (=HS 1804+6753). Fiedler, Barwig \& Mantel (1997) measured $K_{\mathrm{W}}$ and $K_{\mathrm{R}}$, and obtained $i$ from the geometry of the white dwarf and bright spot eclipses. However, the radial velocity curve of the $\mathrm{H} \alpha$ emission line is out of phase by $\sim 0.2$, and the value of $K_{\mathrm{W}}$ determined is therefore unreliable. We have recomputed the system parameters using a Monte Carlo simulation with the values of $K_{\mathrm{R}}$ and $V \sin i$ derived by Billington, Marsh \& Dhillon (1996) and $\Delta \phi_{1 / 2}$ derived by Fiedler et al. See Appendix C.

(xvii) EM Cyg. This double-lined dwarf nova has glancing eclipses, and has had the radial velocities of both components measured by Stover, Robinson \& Nather (1981). Unusually, the secondary star is more massive than the white dwarf. This means that EM Cyg lies outside the $\left(M_{2}, q\right)$ range of thermal and dynamical stability of mass transfer, assuming standard properties of Population I stars. These are, however, sensitive to metallicity, opacities and convection theory used to compute the models. The fact that EM Cyg is observed to be in a moderate state of mass transfer means the star is probably not unstable.

(xviii) AC Cnc. Another eclipsing double-lined system (Schlegel, Kaitchuck \& Honeycutt 1984), also with $q>1$.

(xix) V363 Aur (Lanning 10). Schlegel, Honeycutt \& Kaitchuck (1986) obtained the mass and radius of the secondary star through measurements of $K_{\mathrm{R}}$ and $K_{\mathrm{W}}$, and estimated $i$ using $\Delta \phi_{1 / 2}$.

(xx) BT Mon. Smith et al. (1998) measured $K_{\mathrm{R}}$ from the weak secondary star absorption lines using skew mapping, $K_{\mathrm{W}}, V \sin i$ and $\Delta \phi_{1 / 2}$ to obtain a full set of system parameters using a Monte Carlo simulation.

(xxi) AE Aqr. Casares et al. (1996) detected absorption features from the secondary star and obtained measurements of $K_{\mathrm{R}}$ and $V \sin i$. By modelling the way $V \sin i$ changes with orbital phase, they were able to constrain $i$.

(xxii) DX And. Drew et al. (1993) measured $K_{\mathrm{R}}$ and $V \sin i$, and also estimated $K_{\mathrm{W}}$. Hilditch (1995) used ellipsoidal variations to provide an estimate of $i$. We have used this estimate with the measurements of Drew et al. to calculate the mass and radius of the secondary star. We prefer the $q$ determination of Drew et al. over that derived by Bruch et al. (1997), because Bruch et al. used a phase-shifted $K_{\mathrm{W}}$ measurement from poorly wavelength-calibrated data. (xxiii) V616 Mon (A0620-00). Marsh et al. (1994) measured $K_{\mathrm{R}}, V \sin i$ and ellipsoidal variations in the equivalent width of the $\mathrm{H} \alpha$ emission line. We have used their $1 \sigma$ errors. A further constraint on $i$ is given by the grazing eclipses observed by Haswell et al. (1993), giving $i \sim 70^{\circ}$. However, infrared photometry by Shahbaz, Naylor \& Charles (1994a) of the ellipsoidal variations give a lower inclination, $i \sim 40^{\circ}$, which implies a higher mass and radius, $M_{2} \sim 0.6 \mathrm{M}_{\odot}$ and $R_{2} \sim 0.8 \mathrm{R}_{\odot}$.

(xxiv) $Q Z$ Vul $(G S 2000+25)$. Harlaftis, Horne \& Filippenko (1996) measured $V \sin i$ and $K_{\mathrm{R}}$, and combined these measurements with a wide range of allowable values of $i$ to obtain an estimate of the mass of the secondary.

(xxv) GU Mus (Nova Mus 1991, GRS 1124-68). Casares et al. (1997) measured $K_{\mathrm{R}}$ and $V \sin i$ from the secondary absorption lines. $i$ has been estimated from the ellipsoidal variations (Orosz et al. 1996). Using these parameters, we have calculated the mass and radius of the secondary using a Monte Carlo simulation. See Appendix C.

(xxvi) V1033 Sco (Nova Sco 1994, GRO J1655-040). Orosz \& Bailyn (1997) measured $K_{\mathrm{R}}$ spectroscopically and modelled the light curves, which contained substantial ellipsoidal variations, to obtain $q$ and $i$.

(xxvii) V404 Cyg (GS 2023+338) Casares \& Charles (1994) measured $V \sin i$ and $K_{\mathrm{R}}$ spectroscopically. Shahbaz et al. (1994b) modelled the ellipsoidal variations to obtain $i$ and hence the system parameters. See also Shahbaz et al. (1996a).

\section{APPENDIX B: SIMPLE FORMULAE FOR ESTIMATING THE MASS RATIOAND SECONDARY STAR MASS}

While the best method of calculating the masses of primary and secondary stars and the other system parameters in CVs and LMXBs is the Monte Carlo method (e.g. Smith et al. 1998), simple approximations can give fairly precise estimates of $q$ and $M_{2}$.

$K_{\mathrm{R}}$ and $V \sin i$ are respectively given by

$K_{\mathrm{R}}=\frac{2 \pi}{P} \frac{a}{(1+q)} \sin i$

and

$V \sin i=\frac{2 \pi}{P} R_{2} \sin i$.

Combining these two equations gives

$\frac{R_{2}}{a}(1+q)=\frac{V \sin i}{K_{\mathrm{R}}}$,

which, using equation (2), yields a simple cubic formula for approximating $q$,

$q(1+q)^{2}=9.6\left(\frac{V \sin i}{K_{\mathrm{R}}}\right)^{3}$.

Also, the density-period relation given by equation (3) can be used in combination with equation (16) to give a simple estimate of the mass of the secondary in terms of $V \sin i$ and $P$ if $i$ is known roughly

$\left(\frac{M_{2}}{\mathrm{M}_{\odot}}\right)=0.042\left(\frac{V \sin i}{100 \mathrm{~km} \mathrm{~s}^{-1}}\right)^{3} \frac{P(\mathrm{~h})}{\sin ^{3} i}$.

For eclipsing systems, $\sin ^{3} i$ can be approximated as 0.98 without introducing a significant error. This then leads to the mass of the 
Table C1. Results from the Monte Carlo simulations. Parameters in bold face were those input to the Monte Carlo simulator.

\begin{tabular}{lccccc}
\hline & AM Her & BD Pav & EX Dra & DX And & GU Mus \\
\hline$P(\mathrm{hr})$ & $\mathbf{3 . 0 9}$ & $\mathbf{4 . 3 0}$ & $\mathbf{5 . 0 4}$ & $\mathbf{1 0 . 6 0}$ & $\mathbf{1 0 . 3 8}$ \\
$K_{\mathrm{R}}\left(\mathrm{km} \mathrm{s}^{-1}\right)$ & $\mathbf{1 7 9} \pm \mathbf{2}$ & $\mathbf{2 7 8} \pm \mathbf{4}$ & $\mathbf{2 1 0} \pm \mathbf{1 4}$ & $\mathbf{1 0 5 . 8} \pm \mathbf{3 . 8}$ & $\mathbf{4 2 0 . 8} \pm \mathbf{6 . 3}$ \\
$K_{\mathrm{W}}\left(\mathrm{km} \mathrm{s}^{-1}\right)$ & $115 \pm 18$ & $123 \pm 17$ & $176 \pm 19$ & $103 \pm 9$ & $56 \pm 17$ \\
$V \sin i\left(\mathrm{~km} \mathrm{~s}^{-1}\right)$ & $\mathbf{1 0 0} \pm \mathbf{1 0}$ & $\mathbf{1 2 5} \pm \mathbf{1 0}$ & $\mathbf{1 4 0} \pm \mathbf{1 0}$ & $\mathbf{7 9} \pm \mathbf{5}$ & $\mathbf{1 0 6} \pm \mathbf{1 3}$ \\
$i$ & $\mathbf{5 2} \pm \mathbf{5}$ & $73.4 \pm 0.9$ & $82.1 \pm 2.0$ & $\mathbf{4 9} \pm \mathbf{4}$ & $\mathbf{5 9 . 5} \pm \mathbf{5 . 5}$ \\
$\Delta \phi_{1 / 2}$ & - & $\mathbf{0 . 0 4 0} \pm \mathbf{0 . 0 0 6}$ & $\mathbf{0 . 1 1 0 3} \pm \mathbf{0 . 0 0 0 1}$ & - & - \\
$M_{1}\left(\mathrm{M}_{\odot}\right)$ & $0.44 \pm 0.11$ & $0.95 \pm 0.10$ & $0.70 \pm 0.10$ & $0.51 \pm 0.12$ & $6.98 \pm 1.45$ \\
$M_{2}\left(\mathrm{M}_{\odot}\right)$ & $0.29 \pm 0.10$ & $0.43 \pm 0.10$ & $0.59 \pm 0.12$ & $0.50 \pm 0.14$ & $0.94 \pm 0.40$ \\
$R_{2}\left(\mathrm{R}_{\odot}\right)$ & $0.33 \pm 0.04$ & $0.46 \pm 0.04$ & $0.59 \pm 0.04$ & $0.92 \pm 0.08$ & $1.06 \pm 0.15$ \\
$q$ & $0.64 \pm 0.10$ & $0.44 \pm 0.06$ & $0.85 \pm 0.12$ & $0.98 \pm 0.10$ & $0.13 \pm 0.04$ \\
$a\left(\mathrm{R}_{\odot}\right)$ & $0.96 \pm 0.09$ & $1.48 \pm 0.05$ & $1.58 \pm 0.08$ & $2.44 \pm 0.19$ & $4.77 \pm 0.34$ \\
\hline
\end{tabular}

secondary being solely dependent on $V \sin i$ and $P$.

$\left(\frac{\mathrm{M}_{2}}{\mathrm{M}_{\odot}}\right)=0.043\left(\frac{\mathrm{V} \operatorname{sin~i}}{100 \mathrm{~km} \mathrm{~s}^{-1}}\right)^{3} \mathrm{P}(\mathrm{h})$. using Monte Carlo simulations with values of $P, K_{\mathrm{R}}, V \sin i, i$ and $\Delta \phi_{1 / 2}$ recovered from the literature. The method used is similar to that described by Smith et al. (1998). The resulting system parameters are given in Table $\mathrm{C} 1$.

This paper has been typeset from a $\mathrm{T}_{\mathrm{E}} \mathrm{X} / \mathrm{L}^{\mathrm{A}} \mathrm{T}_{\mathrm{E}} \mathrm{X}$ file prepared by the author.

\section{APPENDIX C: RESULTS FROM MONTE CARLO SIMULATIONS}

A number of the secondary star masses and radii have been derived 